Review

\title{
Portable and Affordable Light Source-Based Photoacoustic Tomography
}

\author{
Mithun Kuniyil Ajith Singh ${ }^{1}(\mathbb{D})$ and Wenfeng $\mathrm{Xia}^{2, *}$ \\ 1 Research and Business Development Division, CYBERDYNE INC., Stationsplein 45, A4.004, \\ 3013 AK Rotterdam, The Netherlands; mithun_ajith@cyberdyne.jp \\ 2 School of Biomedical Engineering\& Imaging Sciences, King's College London, King's Health Partners, \\ St Thomas' Hospital, London SE1 7EH, UK \\ * Correspondence: wenfeng.xia@kcl.ac.uk
}

Received: 8 October 2020; Accepted: 28 October 2020; Published: 29 October 2020

\begin{abstract}
Photoacoustic imaging is a hybrid imaging modality that offers the advantages of optical (spectroscopic contrast) and ultrasound imaging (scalable spatial resolution and imaging depth). This promising modality has shown excellent potential in a wide range of preclinical and clinical imaging and sensing applications. Even though photoacoustic imaging technology has matured in research settings, its clinical translation is not happening at the expected pace. One of the main reasons for this is the requirement of bulky and expensive pulsed lasers for excitation. To accelerate the clinical translation of photoacoustic imaging and explore its potential in resource-limited settings, it is of paramount importance to develop portable and affordable light sources that can be used as the excitation light source. In this review, we focus on the following aspects: (1) the basic theory of photoacoustic imaging; (2) inexpensive light sources and different implementations; and (3) important preclinical and clinical applications, demonstrated using affordable light source-based photoacoustics. The main focus will be on laser diodes and light-emitting diodes as they have demonstrated promise in photoacoustic tomography-the key technological developments in these areas will be thoroughly reviewed. We believe that this review will be a useful opus for both the beginners and experts in the field of biomedical photoacoustic imaging.
\end{abstract}

Keywords: photoacoustic imaging; photoacoustic computed tomography; light-emitting diodes; laser diodes

\section{Introduction}

Photoacoustic imaging (PAI) is an emerging biomedical imaging modality that is based on the detection of ultrasound (US) waves generated from tissue in response to the absorption of temporally varying optical energy [1-5]. Typically, in PAI, short-pulsed or temporally modulated light is delivered to a tissue surface, and the light then propagates diffusively through the tissue during which a portion of the optical energy is selectively absorbed by various light absorbing structures, such as endogenous tissue chromophores and exogenous contrast agents [2]. These endogenous tissue chromophores include DNA/RNA, oxy- and deoxy-hemoglobin, lipid, and melanin; exogenous contrast agents include small molecular dyes, organic nanostructures, metal and carbon nanoparticles, and genetically encoded chromophores [3]. The absorption of the time-varying optical energies leads to rapid and subtle local temperature rises, and subsequently, the generation of broadband US waves in the $\mathrm{MHz}$ frequency range. These initial pressure waves propagate outwards and can be received by US detectors at the tissue surface to form images of the absorbing structures. Since optical absorption spectra of common tissue chromophores (hemoglobin, melanin, lipid, etc.) are well known, it is possible to tune the light excitation wavelength and functionally characterize the tissue using multispectral PAI [1]. For example, one can quantitatively detect oxygen saturation inside a blood vessel with high spatial 
and temporal resolution at depths up to a few centimeters, which is unachievable using any other imaging modalities. PAI involves US detection and it is straight forward to implement this technique in conventional US imaging equipment, offering structural, functional, and molecular imaging details in a single image acquisition [6]. PAI is undoubtedly one of the fastest growing research-based medical imaging modalities in recent times. However, one of the key factors hindering the clinical translation of this promising technique is the requirement of bulky and expensive solid-state lasers for tissue illumination [7]. In this review, after covering the principles of PAI, we will focus on affordable light sources (light-emitting diodes and laser diodes) that are being explored as alternative excitation sources for photoacoustic (PA) tomography, demonstrate its applications, and also discuss the advantages and disadvantages of different illumination sources in PAI. To focus more on PA tomography (PAT) using light-emitting diodes (LEDs) and laser diodes (LDs), we exclude developments and applications demonstrating PA microscopy and endoscopy.

\section{Principles of Photoacoustic Imaging}

Optical tissue imaging utilizes spectroscopic features of light-tissue interactions, such as scattering, absorption, and polarization. Since many tissue molecules possess signature optical properties (especially absorption), this interaction is quite powerful in characterizing tissue at functional and molecular levels. Figure 1 shows the optical absorption spectra of prominent tissue chromophores, such as hemoglobin, melanin, lipid, and water. The rich spectroscopic contrast of these molecules and their relations to a spectrum of diseases make optical imaging very useful [1-3]. However, purely light-based imaging techniques suffer from poor resolution at depths larger than a few mm, due to high scattering in the tissue [1]. While in US imaging, the tissue is insonified using sound waves and echoes are used to generate acoustic reflectivity maps [8]. This is one of the most popular medical imaging modalities with several advantages, including a high portability, affordability, accessibility, and spatial and temporal resolution. Even though US imaging offers tissue anatomical information in real time, it can provide insufficient contrast for soft tissues, and insufficient sensitivity for differentiating malignant and benign abnormalities in deep tissue $[6,9]$.

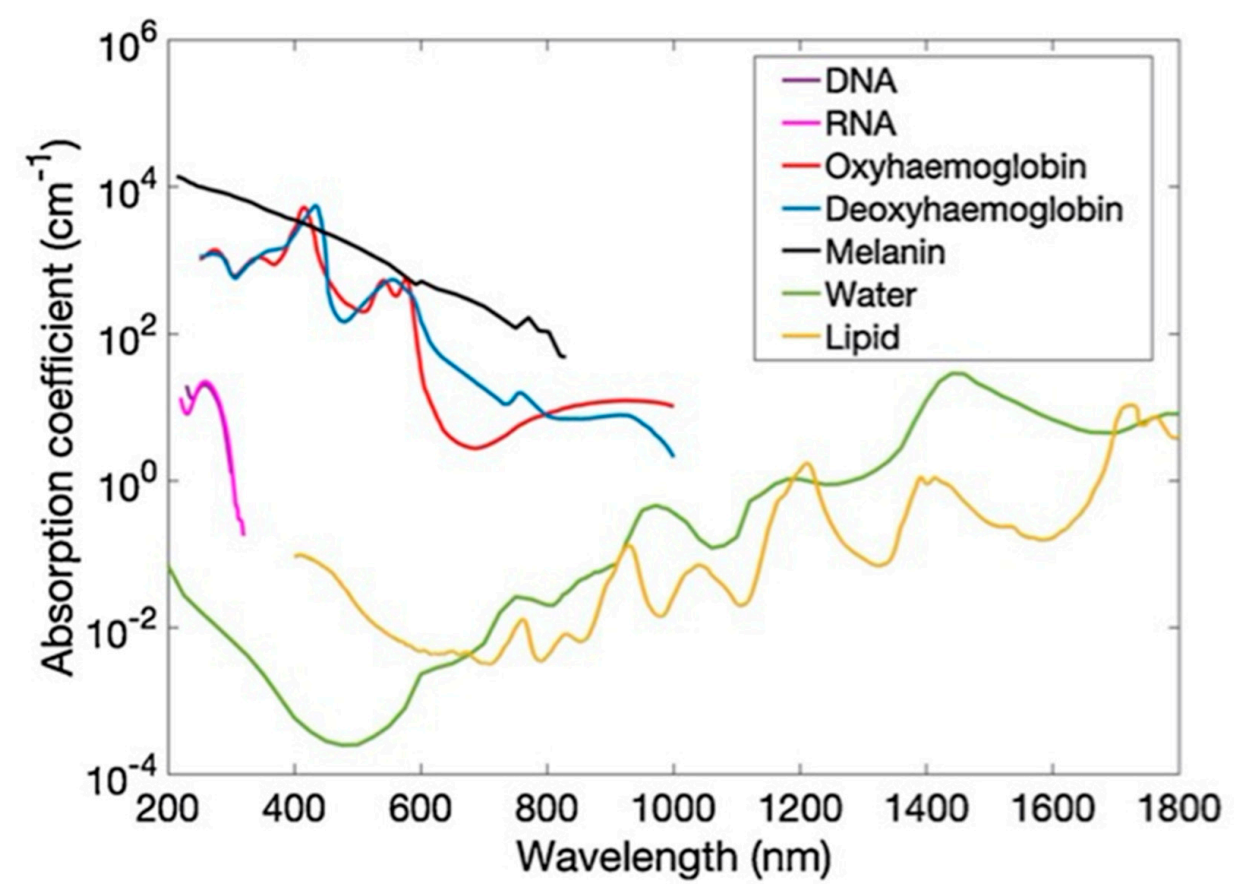

Figure 1. Absorption coefficient spectra ( $\mu$ a) of endogenous tissue chromophores, including DNA, RNA, oxyhemoglobin, deoxyhemoglobin $\left(150 \mathrm{~g} \mathrm{~L}^{-1}\right)$, melanin, water, and lipid. Adapted with permission from T. Zhao, A. E. Desjardins, S. Ourselin, T. Vercauteren, W. Xia, Photoacoustics, Vol.16, Article ID100146, 2019; licensed under a Creative Commons Attribution (CC BY) license. 


\subsection{Generation of Photoacoustic Signals}

The basic idea of the photoacoustic effect was described by Alexander Graham Bell long back in 1880 [10]. When pulsed light is shone on a sample that absorbs a fraction of the incident energy, the optical absorption will result in a temperature rise, leading to thermoelastic expansion of the absorbing object. This sudden pressure rise propagates as a sound wave, which then can be detected using conventional US transducers. By detecting the pressure waves, one can localize their sources (i.e., where the light was absorbed) and obtain important functional and molecular information about the studied sample [1]. The basic idea of PA imaging is schematized in Figure 2.

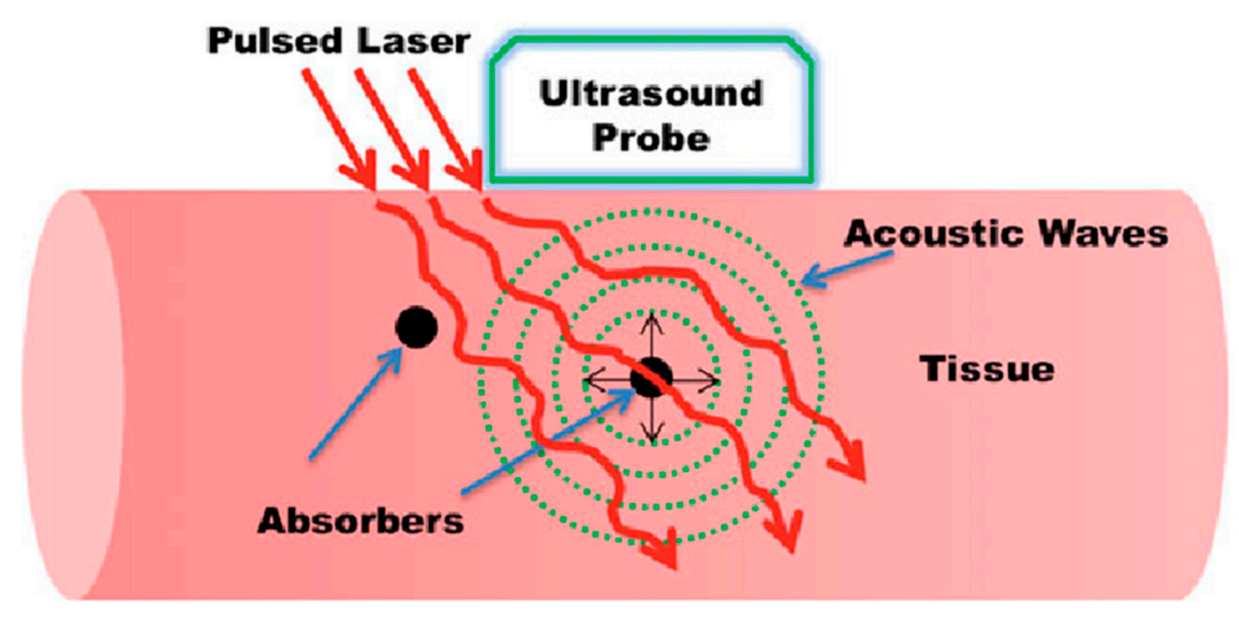

Figure 2. Basic principle of biomedical photoacoustic imaging. Reproduced with permission from Handheld Probe-Based Dual Mode Ultrasound/Photoacoustics for Biomedical Imaging. In: Olivo M., Dinish U. (eds) Frontiers in Biophotonics for Translational Medicine. Progress in Optical Science and Photonics, vol 3. Springer, Singapore (2016). Copyright 2016 Springer, Singapore.

To generate broadband PA signals (sound waves) in the sample efficiently and obtain high-resolution images, the light pulse must be shorter than both the thermal relaxation time $\tau_{t h}$ (thermal confinement) and the stress relaxation time $\tau_{s}$ (stress confinement), respectively, defined by Equations (1) and (2) [1,11].

$$
\tau_{t h}=\frac{d_{c}^{2}}{\alpha_{t h}}
$$

where $d_{c}$ is the characteristic dimension of the structure of interest and $\alpha_{t h}$ is the thermal diffusivity.

$$
\tau_{s}=\frac{d_{c}}{v_{s}}
$$

where $v_{s}$ is the speed of sound in the tissue $(\sim 1540 \mathrm{~m} / \mathrm{s})$.

Let us consider a $15 \mu \mathrm{m}$ optically absorbing structure inside the tissue. In this case, $\tau_{t h}$ and $\tau_{s}$ will be $1.4 \times 10^{-3} \mathrm{~s}$ and $1 \times 10^{-8} \mathrm{~s}$, respectively. Thus, it is clear that the pulse width of the tissue illumination source must be shorter than $10 \mathrm{~ns}$ to spatially resolve this $15 \mu \mathrm{m}$ structure in a PA image. It is also important to note that the detection bandwidth of the US probe also must be high to achieve such high-resolution images, which will be discussed in greater detail later in Section 2.4. The optically induced initial pressure distribution $p_{0}$ can be estimated as

$$
p_{0}(r)=\Gamma \mu_{a}(r) F(r)
$$

where $r$ refers to a spatial location within the heated volume, $\mu_{a}$ is the optical absorption distribution of the absorbing structure, $F$ is the local fluence, $\Gamma$ is the Grüneisen coefficient, and $a$ the dimensionless 
thermodynamic constant that defines the conversion efficiency of heat energy to pressure, and can be expressed as

$$
\Gamma=\beta v_{s}^{2} / C_{p}
$$

where $\beta$ is the isobaric volume expansion coefficient and $C_{p}$ is the specific heat at a constant pressure. It can be seen that $p_{0}$ depends on a number of parameters. Although several studies have shown that $\Gamma$ can be a source of PA contrast in tissue [12], PA image contrast is generally assumed to be dominated by the local optical absorption $\mu_{a}$ and the local fluence $\mathrm{F}$, which is a product of optical absorption and scattering. Considering the strong light attenuation in the tissue, $F$ decreases exponentially with tissue depth; however, optical fluence variations at a given depth in the tissue are usually much smaller compared to those of $\mu_{a}$. As such, PA imaging is often considered as an imaging modality that is based on optical absorption of the tissue; however, it is important to note that the PA image signal is not directly proportional to $\mu_{a}$.

\subsection{Illumination Sources}

The characteristics of the illumination sources mainly define the quality of the PA images and contribute to the high cost of the whole PAI system. PAI systems conventionally use solid-state lasers, such as Q-switched Nd: YAG lasers coupled with optical parametric oscillators (OPO). These lasers usually offer pulse widths in the range of 5-20 ns, which is very much suitable for high-resolution PAI. However, these bulky laser sources are very expensive and are not suitable for use in a clinical setting (for example in an operating room or an outpatient clinic) [13-17]. Recently, there has been significant research in the area of affordable light sources for PAI. Laser diodes (LDs) and light-emitting diodes (LEDs) are the most common PAI illumination sources, which offer high portability, affordability, and energy efficiency [13-17]. Solid-state lasers, LDs, and LEDs have completely different characteristics (pulse width, pulse repetition rate (PRR), optical output, and cost) and have their own advantages and disadvantages. Table 1 shows a comparison of the light sources used in PAI.

Table 1. Comparison of different light sources used in photoacoustic imaging. ${ }^{*}$ Cost includes the driving electronics and may vary based on different features, number of wavelengths, etc. Integration to a US probe may also involve extra development cost. LD, Laser diode; LED: Light-emitting diode; DPSS: Diode-pumped solid-state; PRR, pulse repetition rate. Adapted with permission from Y. Zhu, T. Feng, Q. Cheng, X. Wang, S. Du, N. Sato, J. Yuan and M. Kuniyil Ajith Singh, Sensors, Vol.20, Article ID2484, 2020; licensed under a Creative Commons Attribution (CC BY) license.

\begin{tabular}{|c|c|c|c|c|c|c|}
\hline & Energy (mJ) & PRR (Hz) & $\begin{array}{l}\text { Pulse Width } \\
\text { (ns) }\end{array}$ & Cost * & Advantages & Disadvantages \\
\hline $\begin{array}{l}\text { Solid-state } \\
\text { lasers }\end{array}$ & $5-120$ & $10-200$ & $<10$ & $\$ 70-200 \mathrm{~K}$ & $\begin{array}{l}\text { Powerful, } \sim 5 \mathrm{~cm} \\
\text { penetration depth, } \\
\text { tunable wavelength }\end{array}$ & $\begin{array}{l}\text { Bulky size, eye protection and } \\
\text { laser safe rooms needed }\end{array}$ \\
\hline LD & $0.5-2.5$ & $\sim 1 \mathrm{~K}-6 \mathrm{~K}$ & 30-200 & $\sim \$ 10-25 \mathrm{~K}$ & $\begin{array}{l}\text { Integration in a } \\
\text { handheld probe feasible, } \\
\text { high PRR }\end{array}$ & $\begin{array}{l}\text { Limited penetration depth, eye } \\
\text { protection and laser safe rooms } \\
\text { needed, wavelength tuning not } \\
\text { possible }\end{array}$ \\
\hline LED & 0.2 & $\sim 200-16 \mathrm{~K}$ & $30-100$ & $\$ 10-15 \mathrm{~K}$ & $\begin{array}{l}\text { Integration in a } \\
\text { handheld probe feasible, } \\
\text { high PRR, wide } \\
\text { wavelength range, no } \\
\text { need of laser-safe rooms } \\
\text { and eye-safety goggles }\end{array}$ & $\begin{array}{l}\text { Limited penetration depth, } \\
\text { wavelength tuning not possible }\end{array}$ \\
\hline $\begin{array}{l}\text { Q-switched } \\
\text { DPSS laser }\end{array}$ & 1 & $100 \mathrm{~K}$ & $2-10$ & - & $\begin{array}{l}\text { High PRR, low pulse } \\
\text { width, Reasonably high } \\
\text { optical energy per pulse }\end{array}$ & $\begin{array}{l}\text { Less number of wavelengths } \\
\text { ( } 266 \mathrm{~nm}, 355 \mathrm{~nm}, 532 \mathrm{~nm}, 1064 \\
\mathrm{~nm} \text { ) available and spectral } \\
\text { tuning may be cumbersome }\end{array}$ \\
\hline $\begin{array}{l}\text { High-energy } \\
\text { DPSS laser }\end{array}$ & 200 & 200 & $10-30$ & - & $\begin{array}{l}\text { High optical output per } \\
\text { pulse, reasonably high } \\
\text { PRR }\end{array}$ & $\begin{array}{l}\text { Less number of wavelengths } \\
\text { ( } 266 \mathrm{~nm}, 355 \mathrm{~nm}, 532 \mathrm{~nm}, 1064 \\
\mathrm{~nm} \text { ) available and spectral } \\
\text { tuning may be cumbersome }\end{array}$ \\
\hline
\end{tabular}




\subsection{Optical Absorption}

As shown in Figure 1, intrinsic optical absorbers in the tissue possess the signature absorption characteristics and the spatial distributions of these absorbers can be quantified in PAI with a high spatial resolution and large imaging depths [1]. For example, PAI offers excellent contrast for blood. Since oxyhemoglobin and deoxyhemoglobin have different optical absorption spectra, careful selection of the excitation wavelengths (even two wavelengths with good absorption difference would suffice) can help in obtaining oxygen saturation maps of the tissue with an unprecedented resolution (micro-vasculature level) [16]. Apart from intrinsic absorbers, it is feasible to image exogenous contrast agents by selecting the light wavelengths based on the absorption peak of them [17]. Considering the low absorption of water and high absorption of blood, near infrared (NIR) wavelengths are most commonly used in PAI for achieving higher imaging depths.

\subsection{Ultrasound Propogation and Detection}

The velocity of the US waves in tissue is considered constant at $1540 \mathrm{~m} / \mathrm{s}$ in general. Variations are typically less than $10 \%$, and are usually not accounted for, unless the sample is highly heterogenous. Acoustic scattering in tissue is roughly three orders of magnitude lower than optical scattering and this is the key reason for a higher imaging depth in PAI compared to other optical imaging modalities that rely on optical focusing [1-5]. One important factor to consider acoustically is the attenuation in the tissue, which can be modelled as below.

$$
\alpha=a f^{b}[\mathrm{~dB} / \mathrm{cm}]
$$

where $a$ is a tissue dependent constant, $b$ is a power law factor whose value is usually ranged between 1 and 2 in the soft tissue, and $f$ is the frequency expressed in MHz. In general, for soft tissue, $\alpha$ is considered as $1 \mathrm{~dB} \mathrm{~cm}^{-1} \mathrm{MHz}^{-1}$ on average; it can reach as high as $20 \mathrm{~dB} \mathrm{~cm}^{-1} \mathrm{MHz}^{-1}$ for bone (assuming $b=1$ in both cases). As such, the characteristics of the frequency-dependent acoustic attenuation are similar to those of a low pass filter so that higher frequency signals are attenuated more in tissue. Although the PA signals can be extremely broadband with frequency contents ranging from several tens of $\mathrm{kHz}$ to several tens of $\mathrm{MHz}$, depending on the size of the object, frequency-dependent acoustic attenuation in tissue has limited the maximum frequency content that can arrive at the detector side and thus fundamentally limits the achievable spatial resolution. The spatial resolution of a PA tomography system also depends on other factors, including the frequency bandwidth of the US detector, the active surface area of the detector element, number of detector elements, detector aperture, and image reconstruction algorithms. Moreover, as the acoustic sensitivity of an US detector decreases with the increase in frequency (which largely determines the spatial resolution), there is a trade-off between the imaging depth and spatial resolution. For example, according to a recent study by Xia et al. [18], the measured axial resolution using resolution targets immersed in water for a PAI system with a linear array US probe (central frequency: $9 \mathrm{MHz} ;-6 \mathrm{~dB}$ bandwidth: $77 \%$; active surface area: 5 $\mathrm{mm} \times 0.3 \mathrm{~mm}$; number of element: 128; and detector aperture: $38.4 \mathrm{~mm}$ ) was $0.22 \mathrm{~mm}$ and remained consistent over a depth range of 13 to $36 \mathrm{~mm}$; the lateral resolution depended on the spatial location, ranging from $0.35 \mathrm{~mm}$ to $0.76 \mathrm{~mm}$ over the same depth range.

Linear array piezoelectric probes are commonly used as US detectors for PAI similar to those used for conventional US imaging. As such, real-time interleaved US and PA imaging can be performed with the same US detector and DAQ electronics [6]. US detection in PAI mode is the same as that in the US mode, except that in this case no US transmissions are performed. This dual modality approach offers many advantages involving complementary contrast and easiness in clinical translation. US imaging is a well-accepted imaging modality and it would be easier for clinicians to accept PAI as an additional technique along with conventional US imaging [7]. Typical frequencies for US imaging are in the range of 1-25 MHz. For utilizing the full benefits of PA imaging, it is important to develop new US probes with a higher bandwidth and sensitivity as the PA signals are usually broadband 
and their amplitudes are much lower than the pulse echo signals in US imaging. There have been significant developments in this area recently [6]. Acoustic waves can be detected using single-element transducers (and employing scanning) or using an array of elements as in conventional US probes (planar, cylindrical, or spherical) to enable 2D or 3D imaging.

\subsection{Image Reconstruction}

PAI involves the detection of acoustic signals generated by optical absorption, and image reconstruction strategies are thus stemmed from both optical and US imaging. Basic PA image reconstruction aims to retrieve the spatial distribution of the initial PA pressure or locations of the absorbed optical energy [11]. This is usually termed the acoustic inversion problem and uses various algorithms that originate from the US and sonar world. From the distribution of the initial PA pressure, it is desired to reconstruct a distribution of the optical absorption coefficient, especially when multiple wavelengths are used for PA excitation; however, this is a non-linear problem due to the wavelength-depended optical attenuation of the tissue, which is termed the optical inversion problem. Reconstruction of the optical absorption coefficient is usually termed quantitative PA imaging [11].

There has been extensive research in the area of PA image reconstruction and different methods have been reported. Time reversal, Fourier domain analysis, analytic back-projection, radon transfer, and model-based algorithms are some of the most commonly applied methods. Kuchment et al. reported a detailed review about the different PA-based image reconstruction methods [19]. Based on the type of acoustic detection probes (linear arrays, curved arrays, etc.) and the computational capability, one can choose the right image reconstruction algorithm. Considering the ease in implementation and speed offered, back-projection is the most commonly used image reconstruction technique for processing PA data [1]. Figure 3 illustrates the concept of back-projection algorithms when the PA detection geometry is linear/planar. In this case, each detector element at a specific location records the PA data using the speed of sound (c) and time of flight ( $t$ ), and then the recorded time-resolved signals are back-projected over a spherical surface of radius $\mathrm{R}=\mathrm{ct}$ into the imaging volume [1].

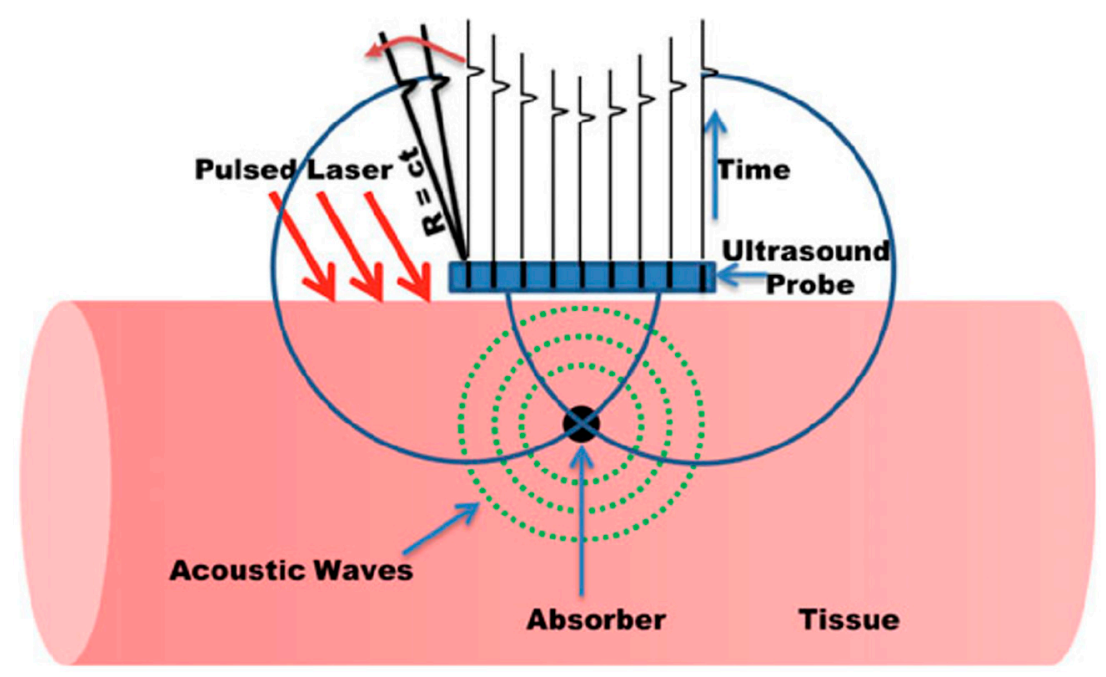

Figure 3. Back-projection PAI reconstruction for a planar detection geometry. Reproduced with permission from Handheld Probe-Based Dual Mode Ultrasound/Photoacoustics for Biomedical Imaging. In: Olivo M., Dinish U. (Eds) Frontiers in Biophotonics for Translational Medicine. Progress in Optical Science and Photonics, volume 3. Springer, Singapore (2016). Copyright 2016 Springer, Singapore.

This method is comparable to the conventional delay-and-sum beamformer, which is the most common algorithm employed in US imaging using phased arrays. It is important to note that a US-based delay-and-sum reconstruction algorithm can be used for PAT data by reducing the time-of-flight to half (PA data traverse half the time in tissue when compared to US signals). This offers the possibility 
to switch between these two image reconstruction modes at a high speed, which is a key requirement in handheld dual-mode PA/US imaging systems. However, the image quality offered by such an approach is not up to the mark. To solve this issue, there have been tremendous developments in the area of frequency domain algorithms, offering superior image quality with computing speeds suitable for systems suitable for real-time imaging [20,21].

\section{LED-Based Photoacoustic Tomography}

In recent years, there have been significant developments in the use of high-power LEDs in biomedical PAI, especially for superficial imaging applications [13,22]. In this section, we review the developments in this area after discussing briefly the characteristics of the LEDs and its advantages and disadvantages.

\subsection{High-Power LEDs Suitable for Photoacoustic Tomography}

An LED is a semiconductor device (P-N junction) that emits light when an electrical current passes through it. Free electrons are the majority charge carriers in N-type semiconductors. On the other hand, holes are the main charge carriers in P-type semiconductors. A P-N junction is formed when the $\mathrm{N}$-type and P-type semiconductors are joined together. When a voltage is applied across the $\mathrm{P}-\mathrm{N}$ junction diode (forward bias), holes (from P-substrate) and electrons (from N-substrate) move towards the junction, resulting in plenty of electrons and holes in the junction region. This fosters a strong electron-hole radiative recombination and consequent emission of photons. In case of LEDs, the radiative recombination process is dependent on spontaneous emission in which electrons in a high energy state $\left(E_{2}\right)$ move down to a lower energy state $\left(E_{1}\right)$ and combine with the available holes. The difference in energy between these two states $\left(E_{g}=E_{2}-E_{1}\right)$ results in spontaneous emission of photons in random directions [23].

\subsection{High-Power LEDs-Technical Aspects}

\subsubsection{Emission Wavelength}

When considering the biomedical imaging applications, a key advantage of LEDs over LDs is the availability of a wide range of wavelengths. As shown in Figure 4, LEDs are available even in the visible wavelengths in which the optical absorption of hemoglobin is quite high [23].

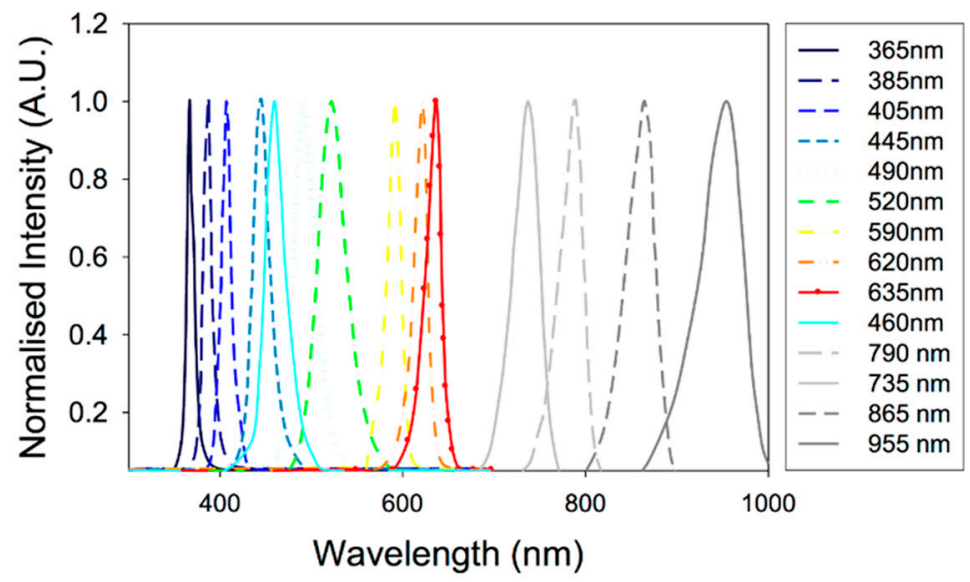

Figure 4. Optical spectra for a range of commercially available LEDs. Reproduced with permission from T. J. Allen and P. C. Beard, Biomedical Optics Express, Vol.7, Article ID1260, 2016; licensed under a Creative Commons Attribution (CC BY) license. 
Materials used for designing the semiconductor (P-N junction) and its energy bandgap ( $\left.\mathrm{E}_{\mathrm{g}}\right)$ are the factors that define the emission wavelength of the LEDs. Thus, one can tune the emission wavelength (visible to NIR range suitable for PAI) of LEDs by carefully selecting the semiconductor material [23]. For example, NIR wavelengths, the most suitable wavelengths for deep-tissue PAI can be developed by using aluminum gallium arsenide (AlxGa1-xAs). By changing the mole fraction (x) of the aluminum $(\mathrm{Al})$ in AlxGa1-xAs, it is straightforward to tune the wavelength range from $624 \mathrm{~nm}$ to $920 \mathrm{~nm}$. For obtaining wavelengths in the visible range $(570-650 \mathrm{~nm})$, aluminum gallium indium phosphide ((AlxGa1-x)0.5In0.5P) is the right candidate for the semiconductor material. In this case, by increasing the mole fraction $(\mathrm{x})$ of the aluminum $(\mathrm{Al})$, one can reduce the emission wavelength. For even shorter wavelengths $(440-550 \mathrm{~nm})$, it is recommended to use indium gallium nitride (InGaN) as the semiconductor material. An increase in indium (In) will result in shifts of emission from shorter to longer wavelengths. The materials used to design LEDs with different wavelengths are summarized in Table 2. With the availability in a wide range of wavelengths, LED illumination is very well suited for multispectral PAI [22].

Table 2. Summary of the materials used to design LEDs with different wavelengths.

\begin{tabular}{cccc}
\hline Wavelength $(\mathrm{nm})$ & $440-550$ & $570-650$ & $624-920$ \\
\hline Material & InGaN & AlGaInP & AlGaAs \\
\hline
\end{tabular}

\subsubsection{Overdriving LEDs}

LEDs are designed to be used for continuous wave (CW) operation and its rated current is valid in this mode. However, it is possible to operate LEDs in the pulsed mode by driving it with higher current pulses of lower duty cycles (less than $<0.1 \%$ ). This way, one can safely increase the optical output and use these affordable devices for PAI [22,24]. Even though LED manufacturers do not provide specifications for pulsed operation, recent studies have demonstrated that it is safe to drive LEDs using a higher current (ten times their rated current) at a duty cycle lower than $0.1 \%$ without any noticeable damage [24].

To operate LEDs in the pulsed mode, special electronic drivers are required. Pulsed LED drivers are most commonly composed of a capacitor, which is used as a storage element that discharges through the LED when a fast-switching device (metal oxide semiconductor field-effect transistors (MOSFETs) are used commonly) is activated. Figure 5 shows a schematic of a typical LED driver.

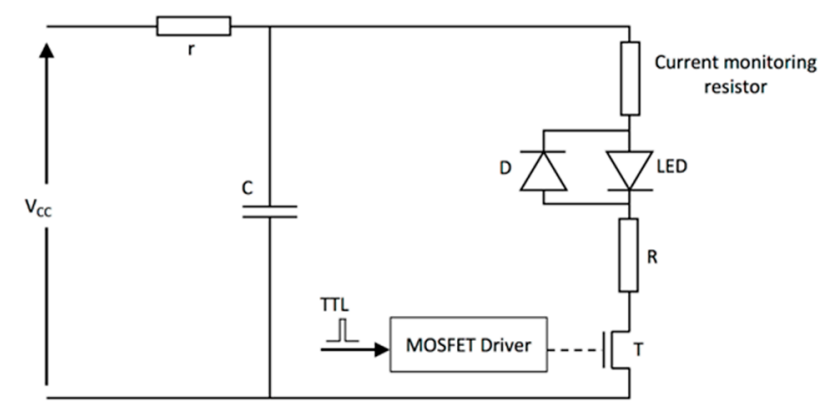

Figure 5. Schematic of a typical LED driver for the pulse operation mode. Vcc is the voltage provided by the power supply, $\mathrm{T}$ is the transistor used to switch the LED on and off, $\mathrm{C}$ is the storage capacitor, $\mathrm{R}$ is the limiting resistor, $\mathrm{r}$ is the charging resistor, $\mathrm{D}$ is a diode, and MOSFET is a metal oxide semiconductor field-effect transistor. Reprinted with permission from High-Power Light Emitting Diodes; An Alternative Excitation Source for Photoacoustic Tomography. In: Kuniyil Ajith Singh M. (eds) LED-Based Photoacoustic Imaging. Progress in Optical Science and Photonics, vol 7. Springer, Singapore. Copyright 2020 Springer, Singapore. 


\subsubsection{Optical Output Power}

In its default continuous wave mode, typical high-power LEDs can provide optical output up to a few watts, which is dependent on the wavelength and also the size of the elements [24]. It is essential to use the LED elements within its allowed current rating (usually around $1 \mathrm{~A}$ ). For avoiding heating issues, high power LED elements are usually mounted on heatsinks. However, it is critical to consider the amount of heat generated, especially when the goal is to integrate a light source and US probe in a single housing for handheld PA and US imaging. Photographs of two representative high-power LEDs are shown in Figure 6, where Figure 6a shows a device with a large emitting area $\left(9 \mathrm{~mm}^{2}\right)$ and Figure $6 \mathrm{~b}$ shows a multi-wavelength device composed of 4 LED elements, each with a smaller emitting area $\left(1 \mathrm{~mm}^{2}\right)$ and at a different wavelength and mounted on a metal-core printed circuit (MCPC) board for efficient heat removal.

a)

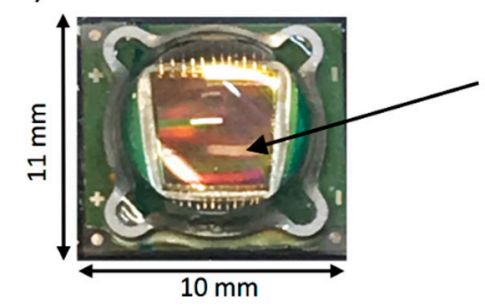

b)

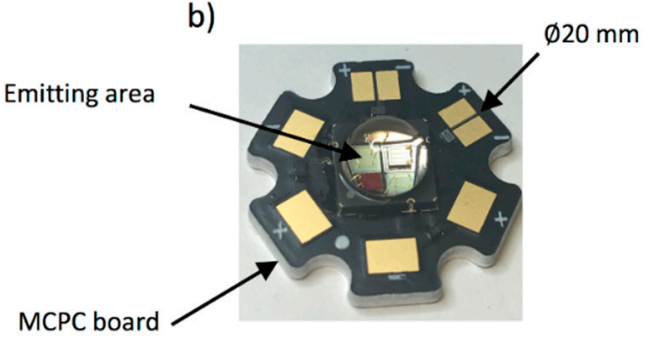

Figure 6. Photographs of high-power LEDs: (a) high-power LED (SST-90) with an emitting area of $9 \mathrm{~mm}^{2}$; (b) high-power multi-wavelength LED (LZ4-00MC00, LedEngin, Inc., California, USA), composed of 4 LEDs emitting at 452,520,520, and $618 \mathrm{~nm}$, each with a $1 \mathrm{~mm}^{2}$ emitting area and mounted on a metal-core printed circuit (MCPC) board. These devices are encapsulated in spherical glass lenses. Reprinted with permission from High-Power Light Emitting Diodes; An Alternative Excitation Source for Photoacoustic Tomography. In: Kuniyil Ajith Singh M. (Eds) LED-Based Photoacoustic Imaging. Progress in Optical Science and Photonics, vol 7. Springer, Singapore. Copyright 2020 Springer, Singapore.

In recent years, the possibility of using multiple LED elements as an array have also been explored to generate a higher optical output required for PAT. Figure 7 shows a photograph of an LED array with $750 \mathrm{~nm}$ and $850 \mathrm{~nm}$ LED elements arranged in an interleaved manner (left panel, 36 elements per row, 4 rows in total) and also the integrated PA and US probe in which the LED arrays are fixed on a conventional linear array US probe (right panel). These high-power LED arrays are commercialized by CYBERDYNE INC (Tsukuba, Japan) for research use [25].
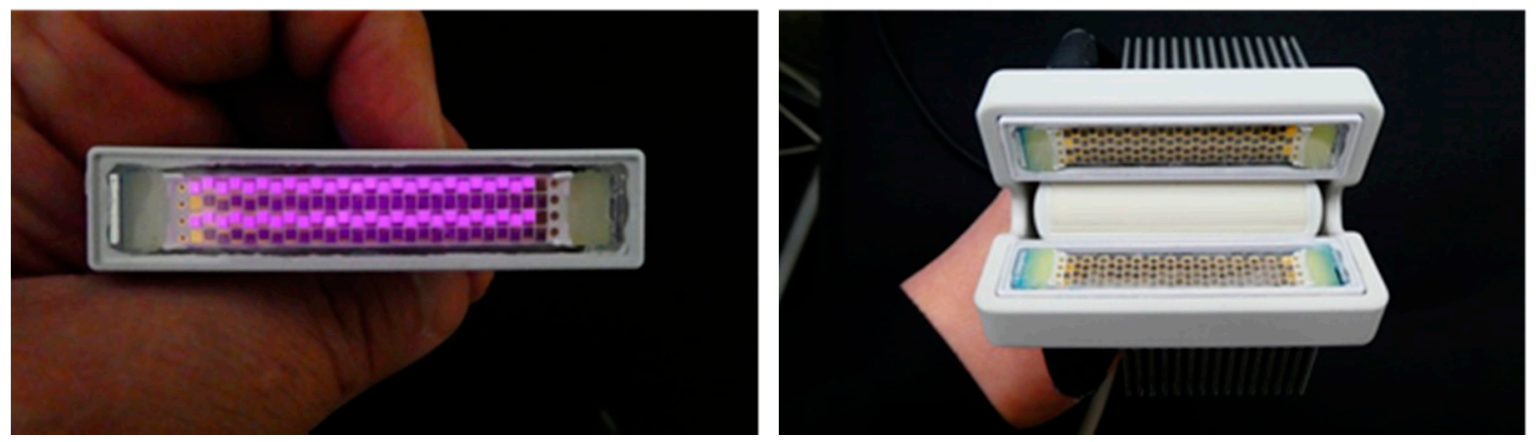

Figure 7. Photograph of an LED array developed by CYBERDYNE INC with four rows of LED elements, in which Rows 1 and 3 are $850 \mathrm{~nm}$ elements, and Rows 2 and 4 are $750 \mathrm{~nm}$ elements (left). In this picture, the $850 \mathrm{~nm}$ elements are activated and captured using an IR camera. Photograph of a LED-based PA/US probe developed by CYBERDYNE INC, in which two LED arrays $(750 / 850 \mathrm{~nm})$ are placed on both sides of a linear array US probe $(7 \mathrm{MHz})$ (right). 


\subsubsection{Pulse Repetition Rate (PRR)}

The typical PRR of conventional solid-state lasers are in the range of 10-200 Hz. For obtaining good signal-to-noise ratios (SNRs) in deep tissue PAI, it is usually required to perform signal averaging over multiple imaging frames, leading to low frame rates in a solid-state laser-based PAI system. On the other hand, PRRs of LEDs are in the $\mathrm{KHz}$ range and this will help to average more frames to improve SNR, without compromising the temporal resolution. Moreover, the large PRR makes high-speed PAT a possibility. LED arrays at $850 \mathrm{~nm}$ with a PRR of $16 \mathrm{KHz}$ have been reported by Zhu et al. recently and they demonstrated dynamic PAI using commercially available LED arrays and conventional linear array US probes [26].

\subsubsection{Pulse Width}

The pulse width of LEDs are tens of nanoseconds (30-100 ns), whereas that of the solid-state lasers is usually less than ten nanoseconds. The temporal pulse width imposes a limit on the spatial resolution of the PAI systems [1]. For example, if an LED element with a $70 \mathrm{~ns}$ pulse width is used as the excitation source, the finest spatial resolution that is potentially achievable can be roughly estimated as $105 \mu \mathrm{m}(=70 \mathrm{~ns} \times 1500 \mu \mathrm{m} / \mu \mathrm{s})$. However, conventional US probes ( $5-7 \mathrm{MHz}$ probes) have reception bandwidth limits and thus further limits its axial resolution to approximately 200-300 $\mu \mathrm{m}$. One may generate broad bandwidth PA signals when the excitation pulse width is low (for example, $5 \mathrm{~ns}$ as in a solid-state laser), but detection sensitivity beyond the US bandwidth is usually very low and the spatial resolution is thus limited by the bandwidth of the US probe. It has been demonstrated that the pulse widths of LEDs are suitable for deep tissue imaging using conventional US probes [13]. A recent study also has shown that tuning the pulse width of the LEDs is feasible and this would be helpful if transducers with different center frequencies and bandwidths are used for the detection [27].

\subsubsection{Spatial Divergence of LEDs}

Conventional high-power LEDs have larger emission angles when compared to other sources. The spatial divergence of commercially available high-power LED arrays (CYBERDYNE INC, Tsukuba, Japan) is approximately $+/-60^{\circ}[28,29]$, which is acceptable in PAT where a relatively large illumination area is usually required. However, because of the large divergence and larger emission area $\left(\sim 1 \mathrm{~mm}^{2}\right)$, it is difficult to collimate the light and couple it to optical fibers for applications like minimally invasive PA imaging [9,30-32]. In terms of eye/skin safety, high spatial divergence in combination with a low optical output makes LEDs a safer alternative to solid-state lasers for non-invasive PAI [20].

\subsection{Technical Developments in LED-Based Photoacoustic Tomography}

The first report on the use of LEDs as an illumination source in PAI was from Jansen in 2011 [33]. In this work, a $627 \mathrm{~nm}$ LED element was used (Luxeon LXHL_PD09), which has been measured to yield approximately $250 \mathrm{~mW}$ of light output when supplied with 1 A DC current. Using a special electronic driver, the LED was supplied with 60-ns current pulses with a peak value of $40 \mathrm{~A}$, resulting in a pulse energy of $400 \mathrm{~nJ}$ per pulse with a pulse width of $60 \mathrm{~ns}$ and a PRR of $200 \mathrm{~Hz}$. Light focusing was performed to generate the fluence required for generating a PA response. To obtain PA signals from a non-realistic gelatin-based phantom, 50,000 A-lines were averaged. Owing to significant developments in the field of solid-state technology, there have been significant developments in LED technology (optical output, PRR, and pulse width), which resulted in the step-by-step development of LED-based PAI technology after 2011.

In 2013, Allen and Beard worked on this further and demonstrated that LEDs can be used as illumination sources in biomedical PA imaging [34]. In this work, they demonstrated the potential of using LEDs as an alternative excitation source in multispectral PA imaging. Recently, the same group achieved an imaging depth of $1.5 \mathrm{~cm}$ in tissue mimicking phantoms when using LEDs as an illumination source in PA imaging [24]. In this work, they obtained an LED output power of $10 \mu \mathrm{J} / \mathrm{pulse}$ 
by overdriving $620 \mathrm{~nm}$ elements and averaging thousands of frames to improve the SNR. Figure 8a shows a schematic of the experimental setup used by them. Figure $8 b$ shows a raw RF signal obtained and $8 \mathrm{c}$ shows a reconstructed image. With the same PRR of $200 \mathrm{~Hz}$, as in the previous study, they achieved a frame rate that is 1000 times better, thanks to the higher optical output achieved using a MOSFET-based electronic driver.

a)
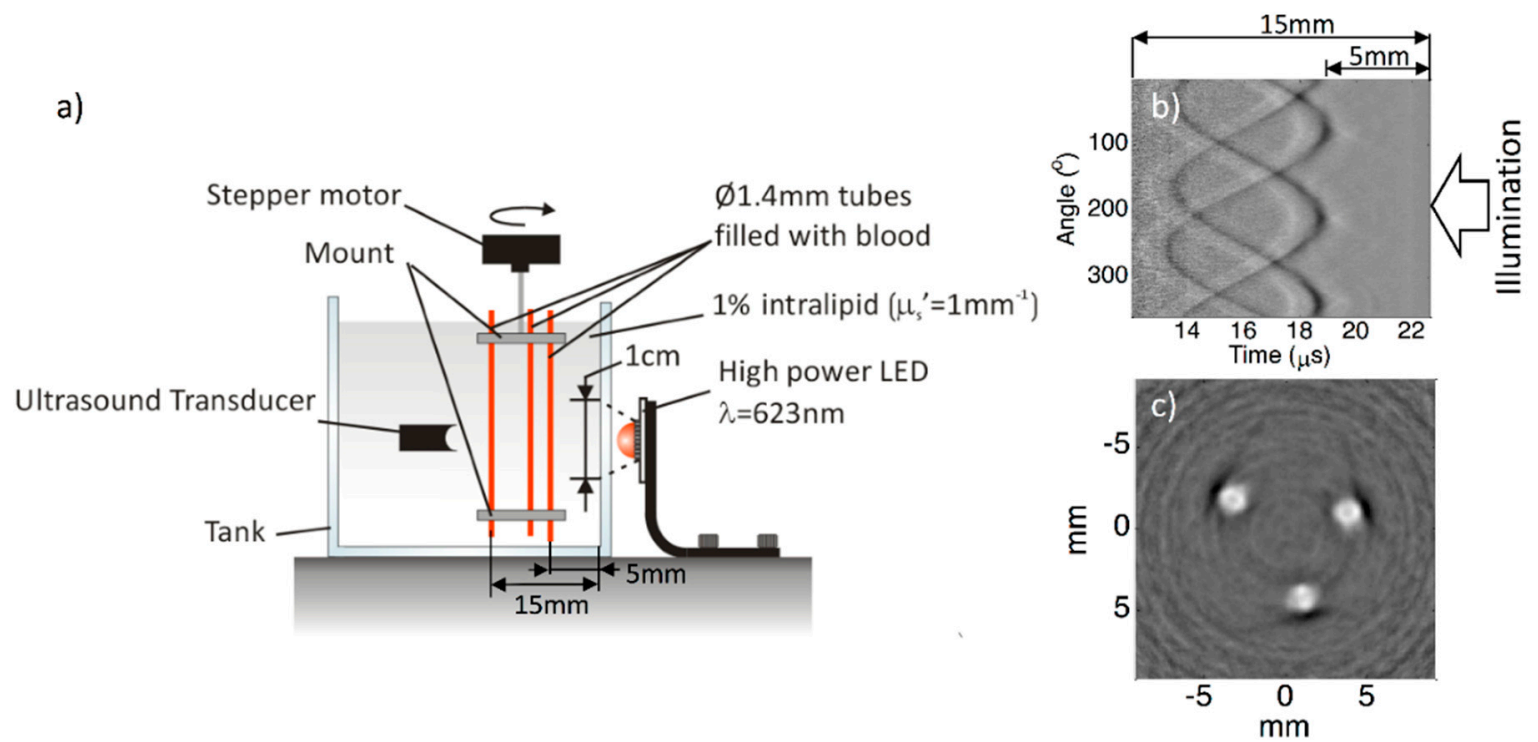

Figure 8. (a) Experimental setup; (b) RF PA signals of three $1.4 \mathrm{~mm}$ tubes filled with human blood immersed in water mixed with intralipid; (c) final reconstructed PA image. Optical output energy attained $=9 \mu \mathrm{J}$. Number of frames averaged: 5000. Reproduced with permission from T. J. Allen and P. C. Beard, Biomedical Optics Express, Vol.7, Article ID1260, 2016; licensed under a Creative Commons Attribution (CC BY) license.

Although these pioneering works demonstrated the feasibility of using LEDs as illumination sources in PAI, no in vivo results have been reported. The main reason behind this is the optical energy of the LEDs, which is magnitudes lower than that of a solid-state laser or even some LDs. In 2015, Agano et al. demonstrated that it was feasible to combine hundreds of high-power LED elements in a rectangular package and could be pulsed simultaneously to perform biomedical PAI [35-37]. Using this novel technology, a high-power LED-array-based PA imaging system (AcousticX) was commercialized by a Japanese company (PreXion Corporation, later the technology was acquired by CYBERDYNE, INC, Japan).

With AcousticX, a single LED element provided an output energy of $0.024 \mu \mathrm{J}$ per pulse, with a pulse duration of $70 \mathrm{~ns}$ and 1 A DC current. By developing LED elements with a double stack structure, arranging them in an array, and applying 20 times the rated current, a light output of $200 \mu \mathrm{J}$ per pulse at a wavelength of $850 \mathrm{~nm}$ was achieved. Two of these arrays were kept on both sides of a linear array US probe for performing real-time PA and US imaging. The repetition rate of this first commercial LED-based PAI system was $4 \mathrm{KHz}$.

The system was thoroughly characterized by several research groups for its spatial resolution and imaging depth, and subsequently its capability for functional, structural, and molecular imaging was demonstrated [26,38-42]. Figure 9 shows a photograph of the system and the PA and US images of a human volunteer's wrist, which was acquired in a real-time handheld operation, demonstrating the capability of obtaining a complementary contrast (structural details from the US image and vasculature details from PA image) in a single measurement [18]. 

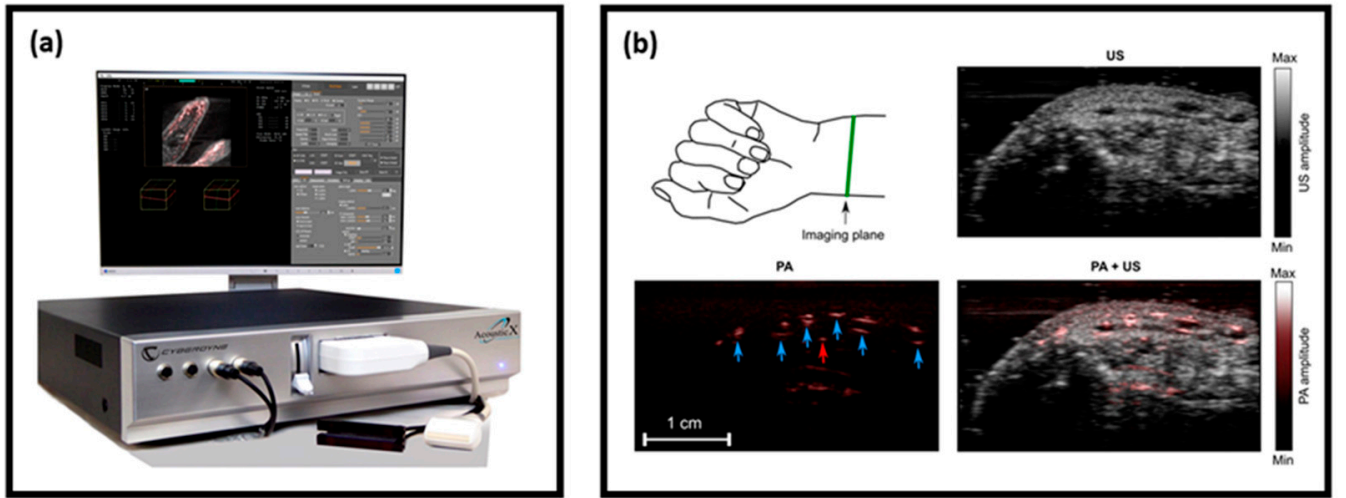

Figure 9. (a) Photograph of the LED-based PA and US imaging system-AcousticX; (b) US (gray colormap), PA (hot colormap), and US/PA overlay cross-section images of a human volunteer's wrist acquired at a frame rate of $10 \mathrm{~Hz}$. Blood vessels are marked using blue arrows in the PA image. Adapted with permission from W. Xia, M. Kuniyil Ajith Singh, E. Maneas, N. Sato, and A. E. Desjardins, Sensors, Vol.18, Article ID1394, licensed under a Creative Commons Attribution (CC BY) license.

In another study [7], utilizing the high temporal resolution of LED-based PAI, it was also possible to scan through an area of interest to generate 3D maps of the vasculature on a human volunteer's foot dorsum, as shown in Figure 10. The total time required to generate such a high-resolution 3D PA image with a large field-of-view was less than $15 \mathrm{~s}$, including scanning and image reconstruction and rendering [7].
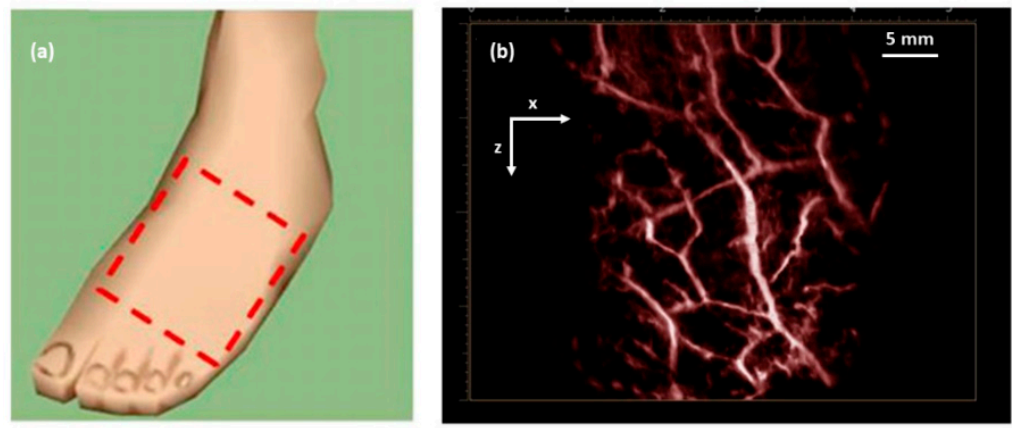

Figure 10. (a) Dashed box showing the imaging area on a human volunteer's foot dorsum; (b) 3D maximum intensity projection PA image of the area marked in (a), clearly visualizing the vasculature network. Adapted with permission from. Reprinted with permission from Clinical Translation of Photoacoustic Imaging-Opportunities and Challenges from an Industry Perspective. In: Kuniyil Ajith Singh M. (Eds) LED-Based Photoacoustic Imaging. Progress in Optical Science and Photonics, vol 7. Springer, Singapore. Copyright 2020 Springer, Singapore.

The system has been recently used extensively by several research groups in a number of studies to explore its potential preclinical and clinical applications [43-51], which are summarized in Table 3. All these reports clearly demonstrated the potential of LED-based PAI in structural, functional, and molecular imaging applications in a preclinical and a clinical setting. Even though the optical output power of the LEDs or LED arrays are not comparable to a solid-state laser, it is encouraging that LED-based PAI is able to consistently achieve an imaging depth of around 5-10 $\mathrm{mm}$. The main reason for this is the high PRR $(\sim 16 \mathrm{KHz})$, which allows averaging over a large number of image frames while maintaining a good temporal resolution; one can easily achieve video rates even after averaging hundreds of frames. Considering the portability, affordability, safety, and ease-of-use, LED-based PAI holds strong potential in clinical translation of PA imaging as an additional modality along with conventional pulse-echo US imaging. 
Table 3. Summary of the preclinical and clinical applications of LED-based PAI. Adapted with permission from Y. Zhu, T. Feng, Q. Cheng, X. Wang, S. Du, N. Sato, J. Yuan and M. Kuniyil Ajith Singh, Sensors, Vol.20, Article ID2484, 2020; licensed under a Creative Commons Attribution (CC BY) license. ICG, indocyanine green.

\begin{tabular}{|c|c|c|c|c|c|}
\hline Target & Application & & $\begin{array}{l}\text { Depth } \\
(\mathrm{mm})\end{array}$ & $\begin{array}{l}\text { Contrast } \\
\text { Agent }\end{array}$ & $\begin{array}{l}\text { Wavelength } \\
\text { (nm) }\end{array}$ \\
\hline $\begin{array}{c}\text { Medical } \\
\text { needles, } \\
\text { Vasculature }\end{array}$ & $\begin{array}{l}\text { Guidance of minimally invasive } \\
\text { procedures with peripheral } \\
\text { tissue targets [18] }\end{array}$ & \multirow{3}{*}{$\begin{array}{l}\text { Phantom and } \\
\text { ex vivo studies }\end{array}$} & 38 & N/A & 850 \\
\hline Vasculature & $\begin{array}{l}\text { Imaging of human placental } \\
\text { vasculature [48] }\end{array}$ & & 7 & N/A & 850 \\
\hline Tumor & $\begin{array}{l}\text { Imaging of intraocular } \\
\text { tumors [26] }\end{array}$ & & 10 & N/A & 850 \\
\hline Vasculature & $\begin{array}{l}\text { Non-invasive monitoring of } \\
\text { angiogenesis [51] }\end{array}$ & \multirow[t]{6}{*}{ Animal in vivo } & 10 & N/A & 850 \\
\hline Ulcer & $\begin{array}{l}\text { Noninvasive imaging of } \\
\text { pressure ulcers [47] }\end{array}$ & & 10 & N/A & 690 \\
\hline $\begin{array}{l}\text { Oxygen } \\
\text { saturation }\end{array}$ & $\begin{array}{l}\text { Oxygen saturation imaging in } \\
\text { rheumatoid arthritis [39] }\end{array}$ & & 5 & N/A & $750 / 850$ \\
\hline Molecular & $\begin{array}{l}\text { Detection and monitoring of } \\
\text { reactive oxygen and nitrogen } \\
\text { species [49] }\end{array}$ & & 10 & CyBA & 850 \\
\hline $\begin{array}{l}\text { Tumor/Contrast } \\
\text { agents }\end{array}$ & $\begin{array}{l}\text { Imaging of tumor using contrast } \\
\text { enhancement [44] }\end{array}$ & & 10 & $\mathrm{NC}$ & 850 \\
\hline $\begin{array}{l}\text { Cells/Contrast } \\
\text { agents }\end{array}$ & $\begin{array}{l}\text { Imaging of molecular-labelled } \\
\text { cells [38] }\end{array}$ & & 10 & DiR & 850 \\
\hline Vasculature & $\begin{array}{l}\text { Imaging of peripheral } \\
\text { microvasculature and } \\
\text { function [26] }\end{array}$ & \multirow[t]{3}{*}{ Healthy human } & 10 & N/A & $690 / 850$ \\
\hline Vasculature & $\begin{array}{l}\text { Simultaneous imaging of veins } \\
\text { and lymphatic vessels [40] }\end{array}$ & & 10 & ICG & $940 / 820$ \\
\hline Finger joints & $\begin{array}{l}\text { Full view tomography of finger } \\
\text { joints [28] }\end{array}$ & & 5 & N/A & 850 \\
\hline Finger joints & $\begin{array}{l}\text { Imaging of inflammatory } \\
\text { arthritis [42] }\end{array}$ & \multirow[t]{2}{*}{ Patient } & 5 & N/A & 850 \\
\hline Skin & Imaging of port wine stain [43] & & 10 & N/A & 850 \\
\hline
\end{tabular}

\section{Laser Diode-Based Photoacoustic Tomography}

Just like LEDs, LDs are also used as an excitation source in PAT, as an alternative to bulky and expensive lasers [52]. With high optical energy (when compared to LEDs) and compactness, pulsed LDs are suitable for performing deep-tissue PAT. In this section, after discussing some of the technical details, specifications, and development of LD-based PAT, we shortly introduce the applications that demonstrate the use of this technique. For the technical aspects, we will focus on the key differences when compared to LEDs.

\subsection{High-Power Pulsed Laser Diodes}

$\mathrm{LD}$ is a semiconductor laser device in which the laser beam is produced at the interface region in a P-I-N (positive-interface-negative) diode region. They are electrically pumped semiconductor laser sources. They convert the input electric energy into light energy, in comparison with conventional lasers in which the input light energy from a flash lamp is converted into laser output [14]. The energy efficiency of these bulky lasers is not high, and this results in an enormous amount of heat generation (water cooling, etc., is thus required, which increases the footprint of the device). On the other hand, the energy efficiency of an LD is very high and thus a minimal amount of heat will be generated. 
In recent years, there have been multiple studies about the development and use of pulsed LDs in PAT $[14,15,53]$.

\subsection{Pulsed Laser Diodes—Technical Aspects}

\subsubsection{Emission Wavelengths}

Conventionally, continuous wave laser diodes are available in a wide range of wavelengths (visible to NIR range). However, pulsed LDs are available only in the NIR range, most probably because of the lower optical energy generated when overdriven with the short pulses required in PAI. In the NIR range, pulsed LDs offer a far higher optical energy when compared to LEDs and thus are ideal sources of illumination in multispectral PAI of deep tissue.

Even though the fundamental process of light generation of LEDs and LDs are the same, LDs generate stimulated emissions and are considered as laser sources with a high spatial coherence. Just as in LEDs, semiconductor materials and their energy band gaps are the deciding factors for the emission wavelengths in LDs. Interestingly, there are some semiconductor compounds in which the bandgap energy can be altered by varying the details of the composition. For example, to achieve shorter wavelengths, one can increase the bandgap energy by increasing the aluminum content (increased $x$ ) in AlxGa1-xAs. Table 4 gives a summary of the semiconductor compounds used for developing LDs with different wavelengths. Even though the visible wavelength range is listed here, these are not commonly available in the pulsed mode.

Table 4. Summary of the materials used to design LDs with different wavelengths.

\begin{tabular}{cccc}
\hline Wavelength $(\mathrm{nm})$ & $630-670$ & $720-850$ & $900-1100$ \\
\hline Material & AlGaInP/GaAs & AlGaAs/GaAs & InGaAs/GaAs \\
\hline
\end{tabular}

\subsubsection{Pulsed Laser Diode Drivers}

Even though continuous wave modulations have also been explored to drive LDs, the most common way is to pulse modulate to generate the short light pulses required for PAI $[15,54]$. A typical LD driver circuit is shown in Figure 11 and the basic idea of driving is similar to that of an LED driver detailed in Section 3.2.2 (charging and discharging of a capacitor).

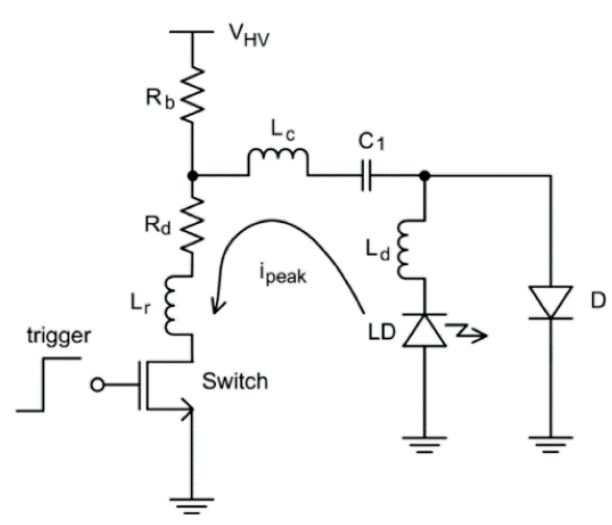

Figure 11. Typical laser driver circuit. Reproduced with permission from H. Zhong, T. Duan, H. Lan, M. Zhou, F. Gao, Sensors, Vol.18, Article ID2264, 2018; licensed under a Creative Commons Attribution (CC BY) license. 
The pulse current flows from the LD to the switching device, as illustrated by the arrow in Figure 11. In order to attain the lower pulse width and boost the peak current, the parasitic inductance $\left(\mathrm{L}_{\mathrm{r}}, \mathrm{L}_{\mathrm{c}}\right.$ and $\mathrm{L}_{\mathrm{d}}$ ) should be as small as possible $[15,55]$. Usually the time constant for these circuits are fixed and tuning of the pulse width is not an option. It is worth mentioning that LEDs on the other hand has already proven to generate light pulses of different pulse widths (30-100 ns). To the best of our knowledge, the most efficient LD driver in the literature so far was reported in 2016, with a size of $40 \times 50 \mathrm{~mm}^{2}$, a PRR up to $10 \mathrm{kHz}$, and a pulse energy of $1.7 \mathrm{~mJ}$ per pulse with a pulse width of $40 \mathrm{~ns}$ [56]. In this work, Canal et al. reported the possibility of integrating a high power LD in its own driver electronic board to save space (Figure 12) and thus opening up the possibility to develop handheld PA probes.
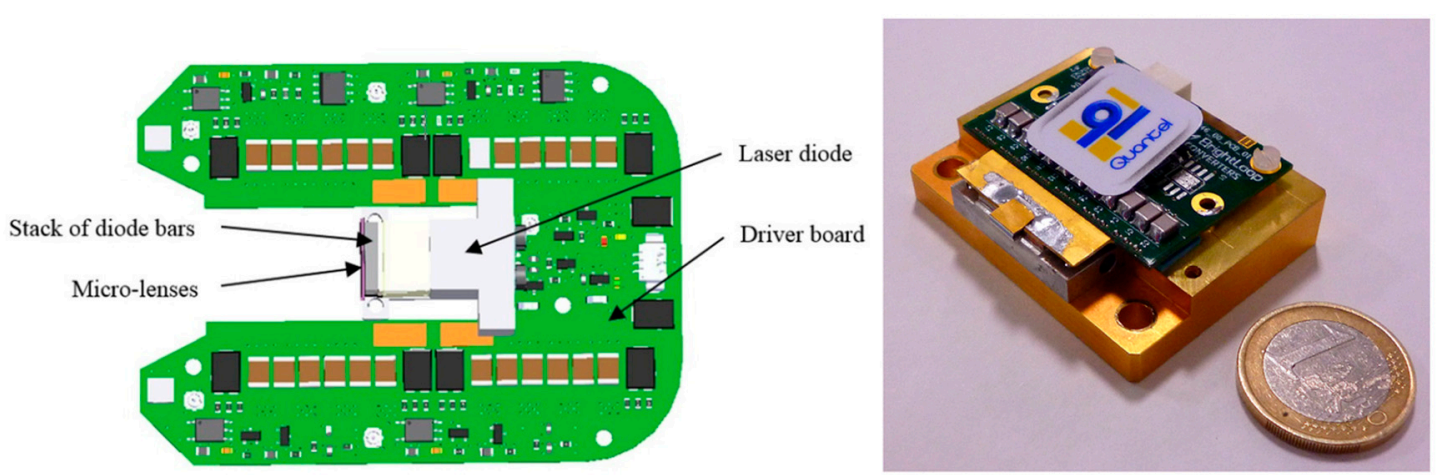

Figure 12. Schematic diagram of the diode laser source showing implementation of the laser diode within the driver board (left). An ultra-short pulse laser source integrating a mini-laser diode providing $1.7 \mathrm{~mJ}$ in pulses as short as $40 \mathrm{~ns}$ from Quantel, France (right). Adapted with permission from Proc. SPIE 9887, Biophotonics: Photonic Solutions for Better Health Care V, 98872B (2016). Copyright 2016 Society of Photo-Optical Instrumentation Engineers (SPIE).

\subsubsection{Optical Output Power}

Conventional pulsed LDs generate laser pulses of relatively low energies over a range of few hundreds of nanojoules to a few microjoules per pulse. However, similar to LEDs, with high PRR LDs $(\sim 10 \mathrm{KHz})$, one can average multiple frames and improve the SNR and imaging depth without much impact on the temporal resolution. Recent developments in semiconductor technology also had positive impacts on the field of LDs. Using diode-stacking technology and ultracompact drivers, a maximum output energy of $1.7 \mathrm{~mJ}$ per pulse was reported in 2016 [56]. Combined with a PRR of $10 \mathrm{KHz}$ and a pulse width of $40 \mathrm{~ns}$, these powerful LDs hold strong potential in deep-tissue high-resolution PAI. Figure 13 shows a schematic comparing diode bars with single and multiple active regions.

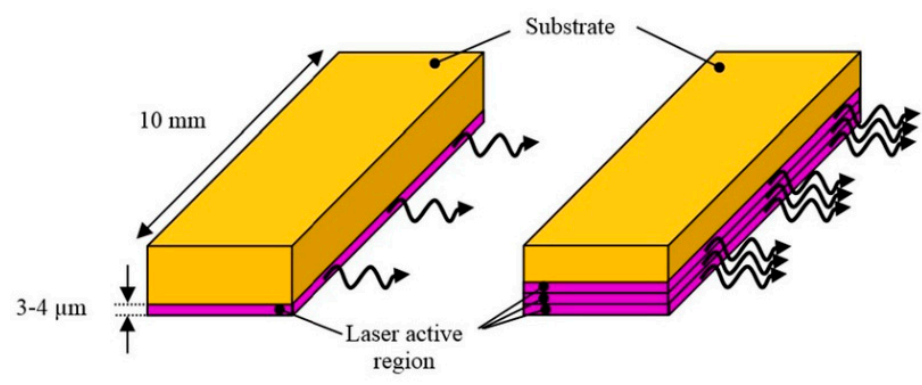

(a)

(b)

Figure 13. Schematic diagram of (a) a standard diode bar and (b) a multiple active region diode bar. Adapted with permission from Proc. SPIE 9887, Biophotonics: Photonic Solutions for Better Health Care V, 98872B (2016). Copyright 2016 Society of Photo-Optical Instrumentation Engineers (SPIE). 


\subsubsection{Pulse Repetition Rate}

Typically, pulsed LDs can be driven at repetition rates of $1-10 \mathrm{KHz}$, which is far higher than the PRR of conventional lasers. To the best of our knowledge, the highest PRR reported for an LD is 10 $\mathrm{KHz}$, which is high enough to be useful for compensating for a low output pulse energy [56]. In this regard, there is flexibility to average multiple frames, maintaining the real-time imaging capability just as in the case of LEDs. Since LDs are coherent light sources as lasers, it is also important to consider the maximum permissible exposure when using higher PRR to ensure light safety, especially in a clinical setting [53].

\subsubsection{Pulse Width}

Pulse widths of commercially available pulsed LDs are in the range of 30-200 ns [53]. This range is higher than that of a conventional solid-state laser, which is capable of delivering $5-10 \mathrm{~ns}$ pulses. However, as discussed before, if stress and thermal confinement can be met, such a low pulse width is not a necessity for deep-tissue PAT. For example, when using $100 \mathrm{~ns}$ LDs as an illumination source, the maximum PA signal frequency expected will be around $10 \mathrm{MHz}$, which is well within the detection bandwidths of US transducers suitable for imaging deep-tissue structures. However, for high-resolution imaging of shallow structures using high-frequency US probes, the pulse widths of the LDs may not be sufficient; also, it is important to mention that the pulse energy of the LD-generated light pulses will drop when the pulse width is reduced. Pulse width-tunable LDs are yet to be reported, which may be because of the technical difficulties in the driver circuit.

\subsubsection{Spatial Divergence of LD}

The spatial divergence of LDs is larger than that of solid-state lasers but smaller than that of LEDs in general, with angles of up to 40 degrees in the axis perpendicular to the diode arrays ("fast axis") and 10 degrees in the parallel axis ("slow axis") [57]. It has been shown that one can efficiently reshape the divergent beam from LDs to be used in an integrated PA and US probe [57]. However, tight optical focusing with these high-power LDs is not feasible due to their multimode nature, which limits their application in optical-resolution PA microscopy.

\subsection{Technical Developments in LD-Based Photoacoustic Tomography}

To the best of our knowledge, the first report on using LDs as a PA illumination source was from Allen and Beard back in 2005 [58]. In this work, they used a pulsed LD (P GAF5S24 from EG\&G; wavelength: $905 \mathrm{~nm}$; and pulse duration: $200 \mathrm{~ns}$ ) driven by a commercially available electronic driver (PCO-7120 from DEI). In this proof-of-concept work, they used a non-realistic phantom (ink-filled cell) and the light was delivered to it using a multimode fiber coupled to the diode. Apart from showing the feasibility of generating PA signals using an LD, they also studied the impact of pulse duration in PAI. In another work immediately after this, Kolkman et al. demonstrated that it was feasible to generate PA signals from superficial blood vessels in a human volunteer [59]. They used a commercially available pulsed laser diode module (iRLS, Laser Components GmbH, Germany) and compared its PAI efficiency with a conventional solid-state laser. The LD used was of $905 \mathrm{~nm}$ wavelength with a pulse duration of $112 \mathrm{~ns}$ and a PRR of $5 \mathrm{kHz}$, and offered a maximum optical energy of $23 \mu \mathrm{J}$ per pulse. Acoustic detection was performed using a double-ring sensor that can generate 1D depth images (A-scans), which then can be used to generate 2D images by rendering multiple A-lines together. Using this setup, for the first time they demonstrated that LDs could image blood vessels in vivo. However, in this encouraging study, the time for one scan (above $3 \mathrm{~min}$ ) and the imaging depth $(1 \mathrm{~mm}$ ) was not optimal.

In late 2016, Allen and Beard improved their $905 \mathrm{~nm}$ LD-based PAT system and showed that it was feasible to image a tissue-mimicking blood vessel phantom effectively [60]. This work confirmed that LDs can very well be an alternative to solid-state lasers for superficial PA imaging applications. In this system, the maximum PRR was $5 \mathrm{kHz}$ and the pulse width was tunable from 50-500 ns. Pulse width 
settings of $65 \mathrm{~ns}$ (pulse energy: $24 \mu \mathrm{J}$ ) and $500 \mathrm{~ns}$ (pulse energy: $184 \mu \mathrm{J}$ ) were used to demonstrate how pulse width affects PA image quality because of stress confinement requirements. A $3.5 \mathrm{MHz}$ single-element PZT focused transducer was used for acoustic detection and 5000 PA frames were averaged to generate one image. Even though slightly slow because of limitations in electronics and data transfer, this work was a solid demonstration of LD-based PAT. Key issues to resolve for using LD-based PAT for in vivo applications were pulse energy and time of acquisition and processing.

After these pioneering works on the use of LDs in PAI, there have been significant developments in the field of combining PAI with conventional pulse-echo US imaging. In this regard, by 2014, Daoudi et al. reported an integrated US and PA imaging probe with a high-power LD (Quantel, France) and all optical components integrated inside one housing along with a commercially available $7 \mathrm{MHz}$ linear array US probe (ESAOTE Europe) [57]. The wavelength of the LD was $805 \mathrm{~nm}$, which emitted $130 \mathrm{~ns}$ pulses with an optical energy of $0.56 \mathrm{~mJ}$ per pulse. The PRR was $10 \mathrm{kHz}$, opening up the possibility to average multiple frames to improve the SNR without sacrificing the frame rate. Figure 14 shows a photograph of this dual-modality PA and US system. As one can see, the probe is quite portable and well suited for a real-time handheld operation in a clinical setting.

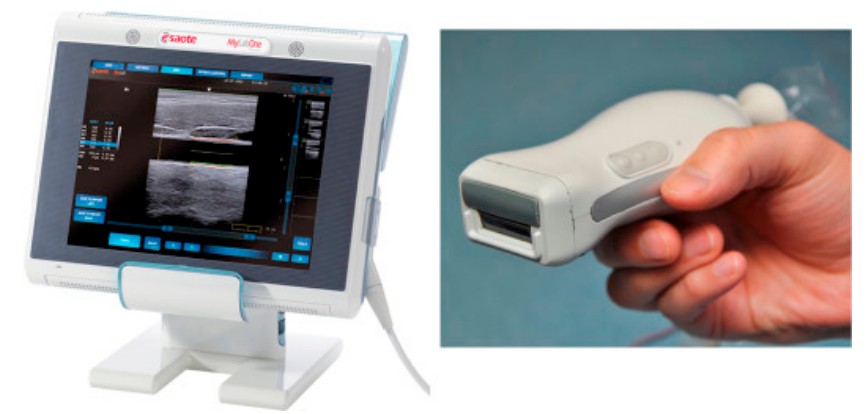

Figure 14. Portable imaging scanner combining photoacoustics and ultrasound. Left is the ultrasound scanner system and right is the picture of the probe integrating the laser module and ultrasound transducer array. Reprinted with permission from K. Daoudi, P.J. van den Berg, O. Rabot, A. Kohl, S. Tisserand, P. Brands, and W. Steenbergen, Optics Express, Vol.22, pp. 26365-26374, 2014; licensed under OSA's "Copyright Transfer and Open Access Publishing Agreement" (OAPA). Copyright 2014 Optical Society of America.

A key feature of this probe design is that all optical components, including the deflecting prism, diffractive optical elements, diode stack, micro-cylindrical lenses, and aluminum cooling rim, were packaged inside a single casing along with a 128-element US probe (Figure 15a). Along with reporting the design of the probe, they performed a detailed characterization of the system and demonstrated the feasibility of in vivo real-time imaging on a human finger. An imaging depth of $4 \mathrm{~mm}$ was achieved in the human finger measurement at a frame rate of $20 \mathrm{~Hz}$ (Figure 15b,c). Higher imaging depths $(10-15 \mathrm{~mm})$ were achieved in phantom studies, but at a higher PRF, which cannot be used in human experiments because of laser safety regulations. Radiant exposure of $1.3 \mathrm{~mJ} / \mathrm{cm}^{2}$ on the skin with an illumination spot size of $18.2 \times 2.3 \mathrm{~mm}^{2}$ was achieved in this prototype, resulting in the possibility of imaging micro-vasculature with unprecedented contrast and resolution. The same system was later used for multiple preclinical applications and early clinical pilot studies [61-66]. Adding multiple diode stacks with different wavelengths can make this system a power tool with excellent structural, functional, and molecular imaging capability. 

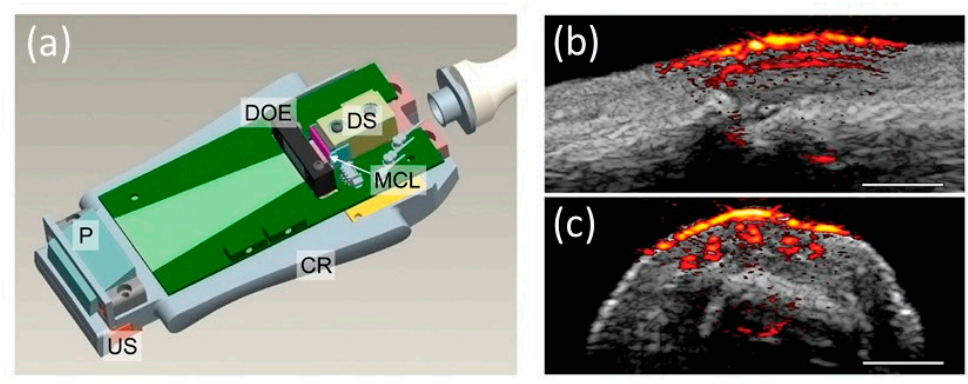

Figure 15. (a) A schematic of the handheld PA and US probe. US: ultrasound array transducer; P: deflecting prism; DOE: diffractive optical elements; DS: diode stack; MCL: micro-cylindrical lenses; CR: aluminum cooling rim. Photoacoustic/ultrasound images of a human proximal interphalangeal joint in the (b) sagittal and (c) transverse planes. Adapted with permission from K. Daoudi, P.J. van den Berg, O. Rabot, A. Kohl, S. Tisserand, P. Brands, and W. Steenbergen, Optics Express, Vol.22, pp. 26365-26374, 2014; licensed under OSA's "Copyright Transfer and Open Access Publishing Agreement" (OAPA). Copyright 2014 Optical Society of America.

In late 2015, Upputuri et al. demonstrated the possibility of PAT using LD excitation and scanning of a single-element US transducer [67]. Using a powerful LD from Quantel (wavelength: $803 \mathrm{~nm}$; pulse energy: $1.4 \mathrm{~mJ}$; and PRR: $7 \mathrm{KHz}$ ) and a conventional single-element US transducer rotating around the object, they achieved to obtain an image every $3 \mathrm{~s}$ and they also demonstrated an imaging depth of 2 $\mathrm{cm}$ in phantom studies, which is commendable. They also compared the results with an OPO-based laser system in similar settings. This system was upgraded by them with multiple single-element transducers and used in multiple preclinical applications recently [68-70]. Figure 16 shows a schematic of the PLD-PAT system designed for in vivo small animal brain imaging and Figure 17 shows images of brain vasculature in a $95 \mathrm{~g}$ female rat acquired non-invasively with PAT at different scan times.

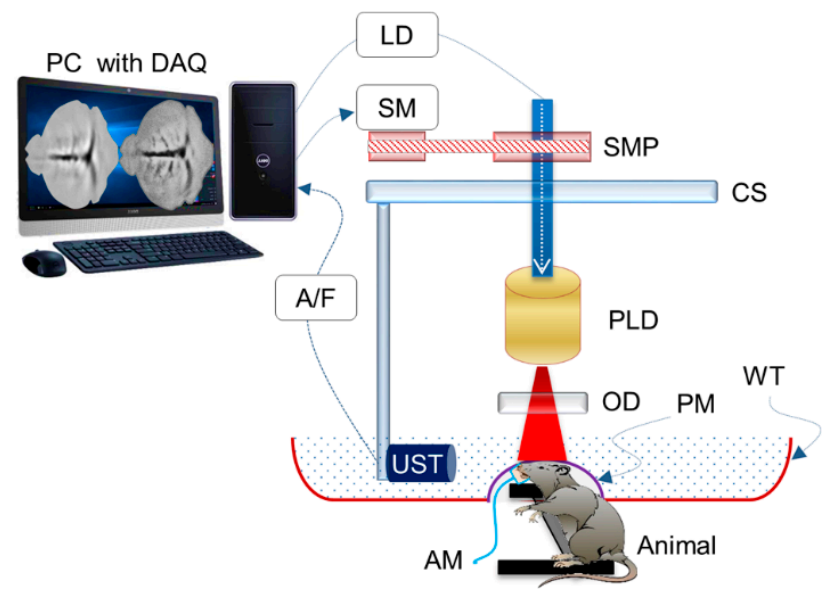

Figure 16. Schematic of the PLD-PAT system for in vivo small animal brain imaging: PLD, pulsed laser diode; OD, optical diffuser; CS, circular scanning plate; SMP, stepper motor pulley unit; UST, ultrasound transducer; A/F, amplifier/filter unit; LD, laser driver unit; SM, stepper motor; PC, personal computer; WT, water tank; DAQ, data acquisition card; AM, anesthesia machine; PM, transparent polythene membrane. Reproduced with permission from P. K. Upputuri and M. Pramanik, Journal of Biomedical Optics, Vol.22, Article ID090501, 2017; licensed under a Creative Commons Attribution (CC BY) license. 

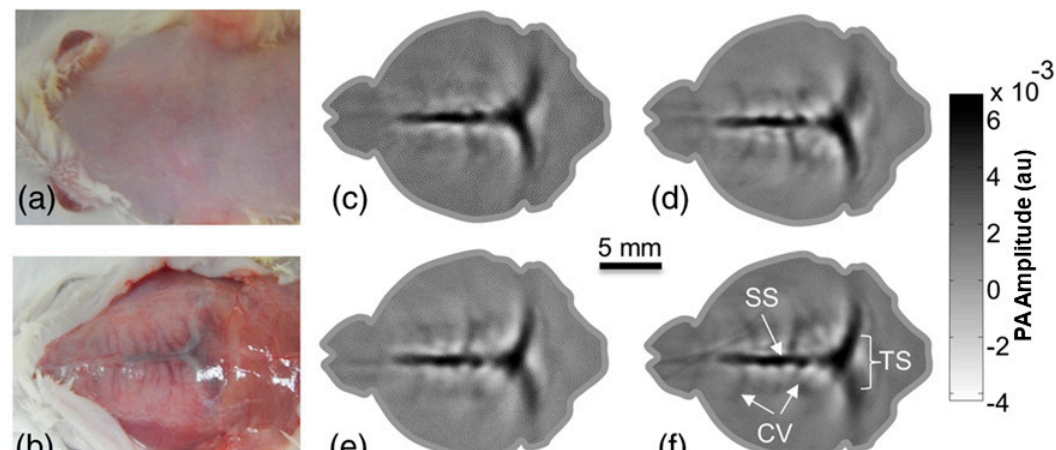

(b)
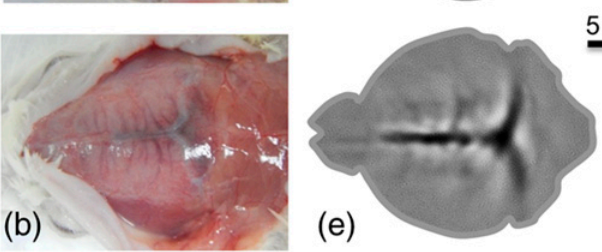

$(\mathrm{f})$

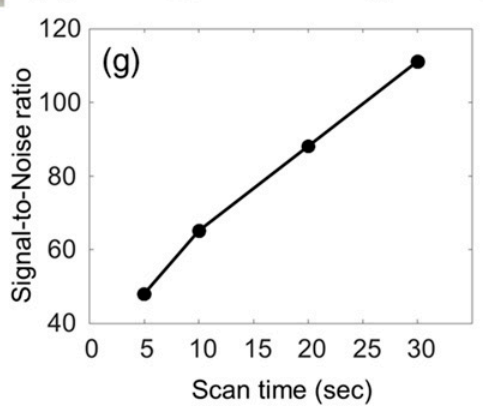

Figure 17. Images of brain vasculature in a $95 \mathrm{~g}$ female rat acquired non-invasively with a laser diode-based photoacoustic tomography system at different scan times: photograph of rat brain before (a) and after (b) removing the scalp. In vivo brain images at a (c) 5-s, (d) 10-s, (e) 20-s, and (f) 30-s scan time. (g) SNR of the in vivo images as a function of scan time. SS, sagittal sinus; TS, transverse sinus; CV, cerebral veins. Adapted with permission from P. K. Upputuri and M. Pramanik, Journal of Biomedical Optics, Vol.22, Article ID090501, 2017; licensed under a Creative Commons Attribution (CC BY) license.

Out of these PLD-PAT works, a recent study from Rajendran et al. [69] is commendable. In this work, the authors used their LD-PAT setup for detecting changes in the sagittal sinus due to intra-cranial hypotension in a rat model. A key advantage of this LD-PAT setup is the cost reduction because of (1) not using US probes with multiple elements; (2) not using multichannel DAQ systems; and (3) replacement of bulky and expensive lasers with LDs. In another recent work from the same group, Upputuri et al. [71] demonstrated a pulsed LD-based PA temperature sensing system for monitoring tissue temperature in real time. The system takes advantages of a laser diode with a high repetition rate $(7000 \mathrm{~Hz})$, a near-infrared wavelength $(803 \mathrm{~nm})$, and a relatively high energy $(1.42$ $\mathrm{mJ} /$ pulse). Results gave a direct confirmation that this LD-based PAI system is capable of providing local temperature information at a high temporal resolution of $1 \mathrm{~ms}$ and high sensitivity of $0.31^{\circ} \mathrm{C}$.

Even though no LD-based PAT system is available commercially, several research studies (Table 5) have validated the potential of LD-based PAT in multiple preclinical and clinical applications, in which encouraging imaging depths were achieved despite low pulse energies when compared with lasers. Pulsed LDs are powerful, portable, and cost-effective, and it is believed that LD-based PAT could be a complementary modality to conventional clinical US imaging [72]. 
Table 5. Summary of the preclinical and clinical applications of LD-based PAI. ICG, indocyanine green.

\begin{tabular}{|c|c|c|c|c|c|}
\hline Target & Application & & $\begin{array}{c}\text { Depth } \\
(\mathrm{mm})\end{array}$ & $\begin{array}{l}\text { Contrast } \\
\text { Agent }\end{array}$ & $\begin{array}{l}\text { Wavelength } \\
\text { (nm) }\end{array}$ \\
\hline Vasculature & $\begin{array}{l}\text { Detection of intraplaque } \\
\text { hemorrhage in carotid } \\
\text { artery [62] }\end{array}$ & Phantom and & 20 & N/A & 808 \\
\hline Vasculature & $\begin{array}{l}\text { Dynamic imaging studies (for } \\
\text { example in cardiovascular } \\
\text { medicine) [73] }\end{array}$ & & 20 & N/A & 803 \\
\hline Red blood cells & $\begin{array}{l}\text { Non-invasive blood flow } \\
\text { imaging [66] }\end{array}$ & & 7 & N/A & 805 \\
\hline Vasculature & Detection of liver fibrosis [64] & Animal in vivo & 5 & N/A & 808 \\
\hline $\begin{array}{c}\text { Cortical } \\
\text { vasculature }\end{array}$ & Brain imaging [68] & & 5 & ICG & 803 \\
\hline $\begin{array}{l}\text { Cerebro-spinal } \\
\text { fluid volume } \\
\text { level }\end{array}$ & $\begin{array}{l}\text { Detection of venous sinus } \\
\text { distension by measuring } \\
\text { intra-cranial hypertension [69] }\end{array}$ & & 5 & N/A & 803 \\
\hline $\begin{array}{l}\text { Vasculature } \\
\text { and perfusion }\end{array}$ & $\begin{array}{l}\text { Vascular/dermal } \\
\text { pathologies [63] } \\
\text { Detection of intraplaque }\end{array}$ & Healthy human & 5 & N/A & 805 \\
\hline Vasculature & $\begin{array}{l}\text { hemorrhage in carotid } \\
\text { artery [65] }\end{array}$ & & 15 & N/A & 808 \\
\hline Finger joints & $\begin{array}{l}\text { Imaging of rheumatoid } \\
\text { arthritis [57] }\end{array}$ & & 5 & N/A & 808 \\
\hline Finger joints & $\begin{array}{l}\text { Imaging of rheumatoid } \\
\text { arthritis [61] }\end{array}$ & Patient & 5 & N/A & 808 \\
\hline
\end{tabular}

\section{Discussion}

In this review, we focused on the basics of PAI and the use of affordable light sources (LEDs and LDs) as illumination sources in PAT. After a short introduction to the physical phenomenon behind biomedical PAI, we elaborated on the key specifications and technological developments in the area of high-power LEDs and LDs and detailed their use as illumination sources in PAT. It is encouraging that even with low optical energies, LED and LD-based PAT systems have already demonstrated their potential in a wide range of functional (oxygen saturation imaging, blood flow imaging, etc.) and molecular imaging applications (tracking contrast agents, pharmacokinetic studies, etc.), thanks to the high PRR (maximum reported PRR for LED: $16 \mathrm{KHz}$; LD: $10 \mathrm{KHz}$ ) and possibility to average over a large number of frames to improve the SNR [13-15]. With these affordable light source-based PAT systems, several in vivo preclinical and clinical pilot studies have reported imaging depths of above 8-10 mm, at frame rates unachievable for conventional laser-based systems [13-15]. Apart from the technical aspects in developing an LED and LD-based PAT system, we also shortly introduced the wide variety of preclinical applications and clinical pilot studies reported using these. Divergence of the LEDs and their large beam size when using an array of LDs make these semiconductors not the optimum choice for PA microscopy applications where a tight light focus is an important requirement [23]. However, there are some reports on the use of LEDs and LDs for microscopic and shallow-depth imaging applications, too [74-79]. We did not include these details in this review as our main focus was on tomographic setups and applications.

PAI is already matured in the research setting and has demonstrated its unprecedented potential in a wide range of biomedical imaging applications. However, the clinical translation of this promising technology is not happening at the expected pace. One of the important reasons for this is the requirement of bulky, slow, and expensive pulsed lasers. In the last decade, there have been significant developments in the semiconductor device technology and use of portable and affordable devices like LEDs and LDs became popular as pulsed light sources in PAT. Q-switched solid-state lasers with 
water-cooling are indeed not an optimal choice for use in a clinical setting because of their sizes, power consumption, costs, and also skin/eye safety aspects (users and patients must wear eye safety glasses and systems must be installed in a laser-safe room). The PRRs of these lasers are also slow and real-time imaging can be challenging, especially when frame averaging is a requirement for improving SNR. Considering the portability, affordability, and dynamic imaging capability aspects, both LEDs and LDs hold strong potential in replacing solid-state lasers, especially for superficial and sub-surface imaging applications, such as in rheumatology, dermatology, and cardiovascular medicine. Handheld US imaging is one of the most popular medical imaging modalities and it is seamless to implement PAT in conventional clinical pulse-echo US equipment. In such handheld systems, integration of LEDs and LDs in a single housing along with a US probe will be a straightforward development and an important aspect to consider in these integrated probes is the mechanism for absorbing the heat generated because of high-speed pulsing. In terms of eye and skin safety, LDs with its coherent nature is similar to that of a conventional laser and the requirements of eye-safety goggles and laser-safe rooms remain important. Even though the fundamental light generation processes for LEDs and LDs are similar, LEDs do not generate stimulated emissions and they possess a broad optical bandwidth and their spatial coherence is low. Considering these factors, LEDs are not practically considered as laser sources and thus could be potentially used as an eye/skin-safe light sources for PAI given the low optical fluence, and the system could be installed in non-laser safe rooms, too, making it an ideal choice for using in a resource-limited clinical setting [13].

Even though LEDs and LDs have shown their potential in biomedical PAT, the pulse energy of these diodes is still a bottleneck when compared to a solid-state laser, and this limits their use only to superficial imaging applications. The maximum pulse energy reported by an LED source (when used in an array form) is $200 \mu \mathrm{J}$ at an $850 \mathrm{~nm}$ wavelength. When two such arrays are used on both sides of an US probe, the total light output will be $400 \mu \mathrm{J}$ per pulse. In such a setting, the maximum imaging depth achieved in vivo was reported to be around $10 \mathrm{~mm}$, with a combined US and PA frame rate above 10 $\mathrm{Hz}$. On the other hand, LDs are more powerful and a pulse energy above $2 \mathrm{~mJ}$ is achievable. However, in an animal/human in vivo situation, so far no LD-based PAI studies has reported an imaging depth above 5-6 mm. An exception is the study from Jaeger et al., in which a carotid artery at a depth of 15 $\mathrm{mm}$ was visualized using an integrated probe with an $808 \mathrm{~nm} \mathrm{LD}$ and a $7 \mathrm{MHz}$ linear array probe. This was achieved by applying an algorithm called DCA (deformation compensated averaging) to reduce the clutter and thereby improving the SNR. It is not straightforward to use a clinical US system for PA detection as highly sensitive and broadband detection with high amplification is very important to achieve high imaging depths. In a nutshell, the maximum imaging depth currently achievable using LED and LED-based PAI systems is less than $2 \mathrm{~cm}$, making it difficult to target deep-tissue imaging applications. Imaging depth in PAI is dependent on several factors, including illumination (type of illumination source, optical output power, wavelength, illumination area, etc.), acoustic detection strategies (sensitivity, directionality, spatial distributions of the US detectors, and noise performance of DAQ, etc.), and image processing and reconstruction algorithms. The conventional solid-state laser-based PAT has already achieved an excellent imaging depth above $6 \mathrm{~cm}$ in phantom studies and 3-4 cm in in vivo clinical pilot studies [80]. It is worth mentioning that a higher imaging depth $(4 \mathrm{~cm})$ was achieved using curved US probes in a computed tomographic setup [81,82]. Using clinical linear array US probes, to the best of our knowledge, an imaging depth above $2 \mathrm{~cm}$ has not been demonstrated in vivo even when using bulky and powerful solid-state lasers as illumination sources. We believe that further advancements in semiconductor device technology and lighting industry in general will improve the pulse energy offered by LDs and LEDs in the near future, thus making these devices well-suited for PAT applications. Theoretically, it is feasible to improve the SNR and thereby imaging depth by increasing the PRR and thus offering more room to increase frame averaging. However, heat generation (especially in fully integrated US/PA probes) and also laser safety will be serious concerns when the PRR is extremely high. It is also well known that when the $\mathrm{N}$ frames are averaged, the SNR will be improved only by $\sqrt{ } \mathrm{N}$. Several US-received side strategies and AI-based methods have also 
been reported for improving the image quality [65,83-91]. We foresee that developments in the area of semiconductor devices and low noise electronics, combined with advanced image reconstruction and enhancement algorithms, will accelerate the clinical translation of affordable light sources based PAI. Since SNR is a key problem to solve, use of novel coded excitation schemes [24,92] and implementation of clutter and reflection artifact reduction algorithms [65,93] also will have a significant impact in clinical translation of LED and LD-based PAT. When compared to OPO-based lasers, one of the key disadvantages of LEDs and LDs is the difficulty in obtaining multiple illumination wavelengths for multispectral PAI [13]. When multiple diode elements with different wavelengths are embedded in an arrayed form, the pulse energy will be further reduced, which then has a serious impact on the imaging depth. Dual-wavelength LED-based PAT for applications like oxygen saturation imaging and differentiation of veins and lymphatic vessels are already reported $[25,40]$. However, in a complex situation with more than two optical absorbers in the tissue, OPO-based laser systems are still preferred because of the wide range of wavelengths available and fast tuning capability.

The pulse duration of the excitation light source is one of the key factors that has an impact on PA signal generation and image quality. It is well known that a short pulse width is important for satisfying the stress confinement criteria and generating broadband PA signals suitable for high-resolution images. Typical pulsed lasers offer a pulse width in the range of 3-10 ns, which then can generate broadband PA signals from the optically absorbing objects. For example, a $3.5 \mathrm{~ns}$ light pulse can generate PA signals with frequencies up to $300 \mathrm{MHz}$, depending on the size of the targets. Such high frequencies from deep tissue will be heavily attenuated and will not reach the US probe. If one has to detect such signals from superficial tissue, it is important to use high frequency US probes with a super-high reception bandwidth. Even though this is feasible technically, applications will be limited to PA microscopy. When using a conventional mid-frequency US probe suitable for deep tissue imaging, the US detection efficiency with frequencies above 12-14 MHz is extremely low [13]. Considering these factors, we believe that such a low pulse width is not an absolute necessity, provided that the stress confinement is met. As discussed before, a very low pulse widths will be only interesting for PA microscopy imaging applications with imaging depth less than 1-2 $\mathrm{mm}$.

In this review, we mainly focused on LEDs and LDs as these are the most common and well-explored affordable PAT light source, especially in the NIR wavelength range, which is the most suitable range of light wavelengths for deep tissue imaging applications $[14,15,53]$. However, in recent years there have been several other reports too on affordable illumination strategies in PAT. In 2016, Wong et al. reported on the use of a single Xenon flash lamp as a light source in PAT [94]. In this promising work, the PRR of the Xenon lamp could be controlled between 10-100 Hz and $3 \mathrm{~mJ}$ of energy was carried in pulses with a $1 \mu$ s pulse width. Using a $0.5 \mathrm{MHz}$ ring-shape detector, the authors demonstrated the feasibility of the imaging tissue, mimicking phantoms (imaging depth: $3.5 \mathrm{~cm}$ ) and a whole mouse body in vivo. Recently, there have been promising reports on the use of diode-pumped solid-state lasers (DPSSLs) as a light source in PAT. PDSSLs are portable and energy efficient, and thus a promising light source for PAI in a resource-limited setting. Wang et al. used a compact high-power DPSSL (Montfort Laser GmbH Inc., Germany) for deep tissue single wavelength in vivo PAT imaging [95]. This laser source has a miniature size of $13.2 \times 14.0 \times 6.5 \mathrm{~cm}^{3}$, a weight of $1.6 \mathrm{~kg}$, and an average power output of $4 \mathrm{~W}$, with a high pulse energy of up to $80 \mathrm{~mJ}$ at the wavelength $1064 \mathrm{~nm}$ with a PRR up to 50 Hz. Using this DPSSL-based system, the authors successfully imaged murine whole-body vascular structures and cardiac functions in vivo, and mapped the arm, palm, and breast vasculatures of living human subjects. One of the key advantages of these DPSSLs are the possibility of fiber coupling and consequent focusing, which is very much important for PA microscopy imaging applications (this is not possible using LED and LEDs). Recently, Jeng et al. reported on the use of a compact 700-900 nm tunable DPSSL with a pulse energy of around $1 \mathrm{~mJ}$ over the range of wavelengths and a PRR of $1 \mathrm{KHz}$ [96]. In this work, the authors demonstrated automatic laser-fluence compensation in spectroscopic PAT and inter-wavelength motion correction using US speckle tracking, which has never been shown before in real-time systems. The $50-\mathrm{Hz}$ video rate PAUS system was demonstrated in vivo 
using a murine model of drug delivery monitoring. As an energy-efficient and portable illumination source, pulsed fiber lasers were also explored as a PAT illumination source. Using a fiber laser emitting at $1060 \mathrm{~nm}$, with a maximum pulse energy of $0.8 \mathrm{~mJ}$ and a Fabry Perot ultrasound scanner, Allen et al. demonstrated the potential of 3D PAT with a realistic blood vessel phantom and the palm vasculature of a human volunteer [97]. Frequency domain PAI systems using continuous-wave modulated laser diodes and LEDs have also been developed recently for several biomedical applications $[98,99]$.

Since short-pulsed light can have a serious impact on the eyes/skin, it is very important to keep the optical pulse energy within the maximum permissible exposure (MPE) limit by following, e.g., the American National Standards Institute (ANSI). Even though the LEDs used in PAI generate pulsed light, they are not considered as laser sources and at present there are no safety standards defined in this regard, to the best of our knowledge. Light from LEDs is optically broad and non-coherent, making it considerably safer to use in a clinical setting as compared to lasers in general (divergence of LEDs is also high, and no safety issues are expected unless the light source is kept very close to the eyes). As a high PRR pulsed laser source, it is very important to consider the MPE limits of the LDs. One may get an initial impression that portable devices like LDs with a low optical power will be completely safe for the eyes and skin. Unfortunately, this is not the case because the light density on the skin will be high when the repetition rate is very high (a large number of light pulses over a period of time). ANSI has clear definitions for this and the safety limits for the skin depend on the light wavelength, pulse width, duration of the exposure, and illumination area [51]. For example, let us consider the case of 800-nm wavelength $(\lambda)$, the MPE limit for the skin over an exposure time $(t)$ of $0.5 \mathrm{~s}$ (assumption) is given by $1.1 \times 10^{(2(\lambda-700) / 1000)} \times \mathrm{t}^{0.25} \mathrm{~J} / \mathrm{cm}^{2}=\sim 1.47 \mathrm{~J} / \mathrm{cm}^{2}$. For an LD with a PRR of $2 \mathrm{KHz}$, the MPE per pulse becomes $\sim 0.735 \mathrm{~mJ} / \mathrm{cm}^{2}$ (1.47/2000). If the LD can deliver optical energy of $1 \mathrm{~mJ}$ per pulse, the light beam must be expanded in such a way that the total area of illumination is about $1.36 \mathrm{~cm}^{2}$ (1/0.735). From this, it is clear that for increasing the PRR for the same exposure time of $0.5 \mathrm{~s}$, the MPE per pulse must be lower than $0.735 \mathrm{~mJ} / \mathrm{cm}^{2}$. In a nutshell, the total pulse energy density will be lower when one increases the PRR, subsequently reducing the possibility of averaging and thus SNR reduction. In clinical PAI, it is of paramount importance to carefully select the pulse energy, PRR, illumination area, and time of exposure for keeping up with the MPE safety limits [52].

\section{Conclusions}

Compact, fast, affordable, and energy-efficient light sources are important requirements for accelerating the clinical translation of PAI. LEDs and LDs fit very well into this category and have been explored extensively in recent years as an illumination source in PAI. This review, which is focused on affordable light source-based PAI, is timely considering the fact that this promising technology is facing an exciting transition from bench to bedside.

In this paper, we first covered the basic theory of PAI, clearly conveying the key advantages of the technique, including optical contrast and acoustic resolution/imaging depth. In the first section, we also shortly introduced the different light sources used in PAI with its specifications, including pulse energy, PRR, pulse width, and cost. After this, the principles of light generation and basic performance characteristics of both LEDs and LDs were discussed with a focus on the advantages and disadvantages when they are used as a light source in PAI. The historical developments (from single-point measurements to in vivo imaging and to commercialization) of both LED- and LD-based PAI systems were also discussed, listing out the key preclinical and clinical application demonstrations.

Even though LEDs and LDs possess some disadvantages, such as low optical energy, lack of spectral tuning capability, and long pulse widths, they are portable, affordable, and energy-efficient light sources. Apart from point-of-care biomedical imaging, LEDs and LDs would be an ideal choice for wearable PA equipment and may find a plethora of applications in the field of therapeutic drug monitoring. Integration of LED- or LD-based PAI to a clinical US scanner will have an easier clinical acceptance when compared to laser-based PAI. We expect that dual-mode US and PA equipment 
utilizing affordable light sources will have a significant impact on bedside diagnostic imaging, accelerating the translation of this technology from research labs to clinics.

Author Contributions: Conceptualization, M.K.A.S. and W.X.; writing-original draft preparation, M.K.A.S.; writing - review and editing, W.X.; supervision, W.X.; project administration, W.X.; funding acquisition, W.X. All authors have read and agreed to the published version of the manuscript.

Funding: This work was supported by the Wellcome Trust [203148/Z/16/Z, WT101957] and the Engineering and Physical Sciences Research Council (EPSRC) [NS/A000027/1, NS/A000049/1].

Conflicts of Interest: Mithun Kuniyil Ajith Singh is employed by CYBERDYNE, INC. The authors have no other financial interests or conflict of interest to disclose. We clarify that in some of the reported studies in this review article, CYBERDYNE, INC as a company was involved in technical developments or design of experiments either directly or through industry-academic collaborative research projects.

\section{References}

1. Beard, P. Biomedical photoacoustic imaging. Interface Focus 2011, 1, 602-631. [CrossRef] [PubMed]

2. Manohar, S.; Razansky, D. Photoacoustics: A historical review. Adv. Opt. Photonics 2016, 8, 586-617. [CrossRef]

3. Wang, L.V. Multiscale photoacoustic microscopy and computed tomography. Nat. Photonics 2009, 3, 503-509. [CrossRef]

4. Omar, M.; Aguirre, J.; Ntziachristos, V. Optoacoustic mesoscopy for biomedicine. Nat. Biomed. Eng. 2019, 3, 354-370. [CrossRef]

5. Wang, L.V.; Yao, L.V.W.J. A practical guide to photoacoustic tomography in the life sciences. Nat. Methods 2016, 13, 627-638. [CrossRef]

6. Singh, M.K.A.; Steenbergen, W.; Manohar, S. Handheld Probe-Based Dual Mode Ultrasound/Photoacoustics for Biomedical Imaging. In Frontiers in Biophotonics for Translational Medicine; Progress in Optical Science and Photonics; Springer: Singapore, 2015; Volume 3, pp. 209-247. [CrossRef]

7. Singh, M.K.A.; Sato, N.; Ichihashi, F.; Sankai, Y. Clinical Translation of Photoacoustic Imaging-Opportunities and Challenges from an Industry Perspective. In LED-Based Photoacoustic Imaging; Progress in Optical Science and Photonics; Springer: Singapore, 2020; Volume 7, pp. 379-393. [CrossRef]

8. Szabo, T.L. Diagnostic Ultrasound Imaging: Inside Out; Elsevier BV: Amsterdam, The Netherlands, 2014.

9. Zhao, T.; Desjardins, A.E.; Ourselin, S.; Vercauteren, T.; Xia, W. Minimally invasive photoacoustic imaging: Current status and future perspectives. Photoacoustics 2019, 16, 100146. [CrossRef]

10. Bell, A.G. On the production and reproduction of sound by light. Am. J. Sci. 1880, 118, 305-324. [CrossRef]

11. Lutzweiler, C.; Razansky, D. Optoacoustic Imaging and Tomography: Reconstruction Approaches and Outstanding Challenges in Image Performance and Quantification. Sensors 2013, 13, 7345-7384. [CrossRef]

12. Tian, C.; Xie, Z.; Fabiilli, M.L.; Wang, X. Imaging and sensing based on dual-pulse nonlinear photoacoustic contrast: A preliminary study on fatty liver. Opt. Lett. 2015, 40, 2253-2256. [CrossRef]

13. Zhu, Y.; Feng, T.; Cheng, Q.; Wang, X.; Du, S.; Sato, N.; Yuan, J.; Singh, M.K.A. Towards Clinical Translation of LED-Based Photoacoustic Imaging: A Review. Sensors 2020, 20, 2484. [CrossRef]

14. Erfanzadeh, M.; Zhu, Q. Photoacoustic imaging with low-cost sources; A review. Photoacoustics 2019, 14, 1-11. [CrossRef] [PubMed]

15. Zhong, H.; Duan, T.; Lan, H.; Zhou, M.; Gao, F. Review of Low-Cost Photoacoustic Sensing and Imaging Based on Laser Diode and Light-Emitting Diode. Sensors 2018, 18, 2264. [CrossRef] [PubMed]

16. Li, M.; Tang, Y.; Yao, J. Photoacoustic tomography of blood oxygenation: A mini review. Photoacoustics 2018, 10, 65-73. [CrossRef]

17. Mallidi, S.; Luke, G.P.; Emelianov, S. Photoacoustic imaging in cancer detection, diagnosis, and treatment guidance. Trends Biotechnol. 2011, 29, 213-221. [CrossRef]

18. Xia, W.; Singh, M.K.A.; Maneas, E.; Sato, N.; Shigeta, Y.; Agano, T.; Ourselin, S.; West, S.J.; Desjardins, A.E. Handheld Real-Time LED-Based Photoacoustic and Ultrasound Imaging System for Accurate Visualization of Clinical Metal Needles and Superficial Vasculature to Guide Minimally Invasive Procedures. Sensors 2018, 18, 1394. [CrossRef]

19. Kuchment, P.; Kunyansky, L. Mathematics of thermoacoustic tomography. Eur. J. Appl. Math. 2008, 19, 191-224. [CrossRef] 
20. Jaeger, M.; Schüpbach, S.; Gertsch, A.; Kitz, M.; Frenz, M. Fourier reconstruction in optoacoustic imaging using truncated regularized inverse k -space interpolation. Inverse Probl. 2007, 23, S51-S63. [CrossRef]

21. Niederhauser, J.J.; Jaeger, M.; Lemor, R.; Weber, P.; Frenz, M. Combined ultrasound and optoacoustic system for real-time high-contrast vascular imaging in vivo. IEEE Trans. Med. Imaging 2005, 24, 436-440. [CrossRef]

22. Singh, M.K.A. LED-Based Photoacoustic Imaging; Springer Nature Singapore Pte Ltd.: Singapore, 2020.

23. Allen, T.J. High-Power Light Emitting Diodes; An Alternative Excitation Source for Photoacoustic Tomography. In LED-Based Photoacoustic Imaging; Progress in Optical Science and Photonics; Springer: Singapore, 2020; Volume 7, pp. 23-43. [CrossRef]

24. Allen, T.J.; Beard, P.C. High power visible light emitting diodes as pulsed excitation sources for biomedical photoacoustics. Biomed. Opt. Express 2016, 7, 1260-1270. [CrossRef]

25. Singh, M.K.A.; Sato, N.; Sankai, Y.; Ichihashi, F. In vivo demonstration of real-time oxygen saturation imaging using a portable and affordable LED-based multispectral photoacoustic and ultrasound imaging system. Photons Plus Ultrasound: Imaging Sens. 2019 2019, 10878, 108785N. [CrossRef]

26. Zhu, Y.; Xu, G.; Yuan, J.; Jo, J.; Gandikota, G.; Demirci, H.; Agano, T.; Sato, N.; Shigeta, Y.; Wang, X. Light Emitting Diodes based Photoacoustic Imaging and Potential Clinical Applications. Sci. Rep. 2018, 8, 1-12. [CrossRef]

27. Singh, M.K.A. Effect of light pulse width on frequency characteristics of photoacoustic signal-An experimental study using a pulse-width tunable LED-based photoacoustic imaging system. Int. J. Eng. Technol. 2018, 7, 4300-4303.

28. Francis, K.J.; Boink, Y.E.; Dantuma, M.; Singh, M.K.A.; Manohar, S.; Steenbergen, W.; Joseph, F.K. Tomographic imaging with an ultrasound and LED-based photoacoustic system. Biomed. Opt. Express 2020, 11, 2152-2165. [CrossRef]

29. Francis, K.J.; Boink, Y.E.; Dantuma, M.; Singh, M.K.A.; Manohar, S.; Steenbergen, W. Light Emitting Diodes Based Photoacoustic and Ultrasound Tomography: Imaging Aspects and Applications. In LED-Based Photoacoustic Imaging; Progress in Optical Science and Photonics; Springer: Singapore, 2020; Volume 7, pp. 245-266. [CrossRef]

30. Xia, W.; Nikitichev, D.I.; Mari, J.M.; West, S.J.; Pratt, R.; David, A.L.; Ourselin, S.; Beard, P.C.; Desjardins, A.E. Performance characteristics of an interventional multispectral photoacoustic imaging system for guiding minimally invasive procedures. J. Biomed. Opt. 2015, 20, 086005. [CrossRef]

31. Mari, J.M.; Xia, W.; West, S.J.; Desjardins, A.E. Interventional multispectral photoacoustic imaging with a clinical ultrasound probe for discriminating nerves and tendons: An ex vivo pilot study. J. Biomed. Opt. 2015, 20, 110503. [CrossRef] [PubMed]

32. Singh, M.K.A.; Parameshwarappa, V.; Hendriksen, E.; Steenbergen, W.; Manohar, S. Photoacoustic-guided focused ultrasound for accurate visualization of brachytherapy seeds with the photoacoustic needle. J. Biomed. Opt. 2016, 21, 120501. [CrossRef] [PubMed]

33. Hansen, R.S. Using high-power light emitting diodes for photoacoustic imaging. SPIE Med. Imaging 2011, 7968, 79680. [CrossRef]

34. Allen, T.J.; Beard, P.C. Light emitting diodes as an excitation source for biomedical photoacoustics. In Proceedings of the Photons Plus Ultrasound: Imaging and Sensing 2013, San Francisco, CA, USA, 4 March 2013; p. 85811F. [CrossRef]

35. Agano, T.; Sato, N.; Nakatsuka, H.; Kitagawa, K.; Hanaoka, T.; Morisono, K.; Shigeta, Y. Comparative experiments of photoacoustic system using laser light source and LED array light source. In Proceedings of the Photons Plus Ultrasound: Imaging and Sensing 2015, San Francisco, CA, USA, 11 March 2015; p. 93233X. [CrossRef]

36. Agano, T.; Sato, N.; Nakatsuka, H.; Kitagawa, K.; Hanaoka, T.; Morisono, K.; Shigeta, Y. Attempts to increase penetration of photoacoustic system using LED array light souce. In Proceedings of the Photons Plus Ultrasound: Imaging and Sensing 2015, San Francisco, CA, USA, 11 March 2015; p. 93233Z. [CrossRef]

37. Agano, T.; Sato, N. Photoacoustic Imaging System using LED light source. In Proceedings of the 2016 Conference on Lasers and Electro-Optics (CLEO), San Jose, CA, USA, 5-10 June 2016; The Optical Society: Washington, DC, USA, 2016; p. ATh3N.5.

38. Hariri, A.; LeMaster, J.; Wang, J.; Jeevarathinam, A.S.; Chao, D.L.; Jokerst, J.V. The characterization of an economic and portable LED-based photoacoustic imaging system to facilitate molecular imaging. Photoacoustics 2018, 9, 10-20. [CrossRef] 
39. Joseph, F.K.; Xavierselvan, M.; Singh, M.K.A.; Mallidi, S.; Van Der Laken, C.; Van De Loo, F.; Steenbergen, W. LED-based photoacoustic imaging for early detection of joint inflammation in rodents: Towards achieving 3Rs in rheumatoid arthritis research. In Proceedings of the Photons Plus Ultrasound: Imaging and Sensing 2020, San Francisco, CA, USA, 17 February 2020; p. 112400M. [CrossRef]

40. Singh, M.K.A.; Agano, T.; Sato, N.; Shigeta, Y.; Uemura, T. Real-time in vivo imaging of human lymphatic system using an LED-based photoacoustic/ultrasound imaging system. In Proceedings of the Photons Plus Ultrasound: Imaging and Sensing 2018, San Francisco, CA, USA, 19 February 2018; p. 1049404. [CrossRef]

41. Singh, M.K.A.; Shigeta, Y.; Hanaoka, T.; Agano, T.; Sato, N. High-speed photoacoustic imaging using an LED-based photoacoustic imaging system. In Proceedings of the Photons Plus Ultrasound: Imaging and Sensing 2018, San Francisco, CA, USA, 19 February 2018; p. 104943N. [CrossRef]

42. Jo, J.; Xu, G.; Zhu, Y.; Burton, M.; Sarazin, J.; Schiopu, E.; Gandikota, G.; Wang, X. Detecting joint inflammation by an LED-based photoacoustic imaging system: A feasibility study. J. Biomed. Opt. 2018, 23, 110501-110504. [CrossRef]

43. Cheng, Q.; Qian, M.; Wang, X.; Zhang, H.; Wang, P.; Wen, L.; Pan, J.; Gao, Y.; Wu, S.; Zhang, M.; et al. Diagnosis and Treatment Monitoring of Port-Wine Stain Using LED-Based Photoacoustics: Theoretical Aspects and First In-Human Clinical Pilot Study. In LED-Based Photoacoustic Imaging; Progress in Optical Science and Photonics; Springer Nature Singapore Pte Ltd.: Singapore, 2020; pp. 351-377.

44. Xavierselvan, M.; Mallidi, S. LED-Based Functional Photoacoustics-Portable and Affordable Solution for Preclinical Cancer Imaging. In LED-Based Photoacoustic Imaging; Progress in Optical Science and Photonics; Springer: Singapore, 2020; Volume 7, pp. 303-319. [CrossRef]

45. Agrawal, S.; Fadden, C.; Dangi, A.; Yang, X.; AlBahrani, H.; Frings, N.; Zadi, S.H.; Kothapalli, S.-R. Light-Emitting-Diode-Based Multispectral Photoacoustic Computed Tomography System. Sensors 2019, 19, 4861. [CrossRef]

46. Francis, K.J.; Booijink, R.; Bansal, R.; Steenbergen, W. Tomographic Ultrasound and LED-Based Photoacoustic System for Preclinical Imaging. Sensors 2020, 20, 2793. [CrossRef]

47. Hariri, A.; Chen, F.; Moore, C.; Jokerst, J.V. Noninvasive staging of pressure ulcers using photoacoustic imaging. Wound Repair Regen. 2019, 27, 488-496. [CrossRef]

48. Maneas, E.; Aughwane, R.; Huynh, N.; Xia, W.; Ansari, R.; Singh, M.K.A.; Hutchinson, J.C.; Sebire, N.J.; Arthurs, O.J.; Deprest, J.; et al. Photoacoustic imaging of the human placental vasculature. J. Biophotonics 2019, 13, e201900167. [CrossRef]

49. Hariri, A.; Zhao, E.; Jeevarathinam, A.S.; LeMaster, J.; Zhang, J.; Jokerst, J.V. Molecular imaging of oxidative stress using an LED-based photoacoustic imaging system. Sci. Rep. 2019, 9, 11378. [CrossRef]

50. Xia, W.; Maneas, E.; Huynh, N.T.; Singh, M.K.A.; Brown, N.M.; Ourselin, S.; Gilbert-Kawai, E.; West, S.J.; Desjardins, A.E. Imaging of human peripheral blood vessels during cuff occlusion with a compact LED-based photoacoustic and ultrasound system. In Proceedings of the Photons Plus Ultrasound: Imaging and Sensing 2019, San Francisco, CA, USA, 27 February 2019; p. 1087804. [CrossRef]

51. Zhu, Y.; Lu, X.; Dong, X.; Yuan, J.; Fabiilli, M.L.; Wang, X. LED-Based Photoacoustic Imaging for Monitoring Angiogenesis in Fibrin Scaffolds. Tissue Eng. Part C Methods 2019, 25, 523-531. [CrossRef]

52. Kalva, S.K.; Pramanik, M. Photoacoustic Tomography with High-Energy Pulsed Laser Diodes; SPIE Spotlight Series Book; SPIE-Intl Soc Optical Eng: Bellingham, WA, USA, 2020; Volume SL56, ISBN 9781510636620. Available online: https://spie.org/Publications/Book/2566566?SSO=1 (accessed on 28 October 2020).

53. Fatima, A.; Kratkiewicz, K.; Manwar, R.; Zafar, M.; Zhang, R.; Huang, B.; Dadashzadeh, N.; Xia, J.; Avanaki, K. (Mohammad) Review of cost reduction methods in photoacoustic computed tomography. Photoacoustics 2019, 15, 100137. [CrossRef]

54. Choi, S.S.S.; Mandelis, A. Review of the state of the art in cardiovascular endoscopy imaging of atherosclerosis using photoacoustic techniques with pulsed and continuous-wave optical excitations. J. Biomed. Opt. 2019, 24, 1-15. [CrossRef]

55. Nissinen, J.; Kostamovaara, J. A 4 a peak current and 2 ns pulse width CMOS laser diode driver for high measurement rate applications. In Proceedings of the 2013 ESSCIRC (ESSCIRC), Bucharest, Romania, 16-20 September 2013; Institute of Electrical and Electronics Engineers (IEEE): Bucharest, Romania, 2013; pp. 355-358.

56. Canal, C.; Laugustin, A.; Kohl, A.; Rabot, O. Portable multiwavelength laser diode source for handheld photoacoustic devices. Biophotonics: Photonic Solutions for Better Health Care V 2016, 9887, 98872. [CrossRef] 
57. Daoudi, K.; Berg, P.V.D.; Rabot, O.; Kohl, A.; Tisserand, S.; Brands, P.; Steenbergen, W. Handheld probe integrating laser diode and ultrasound transducer array for ultrasound/photoacoustic dual modality imaging. Opt. Express 2014, 22, 26365-26374. [CrossRef]

58. Allen, T.J.; Cox, B.T.; Beard, P.C. Generating photoacoustic signals using high-peak power pulsed laser diodes. In Proceedings of the Photons Plus Ultrasound: Imaging and Sensing 2005: The Sixth Conference on Biomedical Thermoacoustics, Optoacoustics, and Acousto-optics, San Jose, CA, USA, 25 April 2005; SPIE-Intl Soc Optical Eng: Bellingham, WA, USA, 2005; Volume 5697, pp. 233-243.

59. Kolkman, R.G.M.; Steenbergen, W.; Van Leeuwen, T.G. In vivo photoacoustic imaging of blood vessels with a pulsed laser diode. Lasers Med Sci. 2006, 21, 134-139. [CrossRef] [PubMed]

60. Allen, T.J.; Beard, P.C. Pulsed near-infrared laser diode excitation system for biomedical photoacoustic imaging. Opt. Lett. 2006, 31, 3462-3464. [CrossRef]

61. Berg, P.J.V.D.; Daoudi, K.; Moens, H.J.B.; Steenbergen, W. Feasibility of photoacoustic/ultrasound imaging of synovitis in finger joints using a point-of-care system. Photoacoustics 2017, 8, 8-14. [CrossRef]

62. Arabul, M.U.; Heres, M.; Rutten, M.C.M.; Van Sambeek, M.R.; Van De Vosse, F.N.; Lopata, R.G.P. Toward the detection of intraplaque hemorrhage in carotid artery lesions using photoacoustic imaging. J. Biomed. Opt. 2016, 22, 41010. [CrossRef]

63. Heres, H.M.; Arabul, M.U.; Rutten, M.C.M.; Van De Vosse, F.N.; Lopata, R.G.P. Visualization of vasculature using a hand-held photoacoustic probe: Phantom and in vivo validation. J. Biomed. Opt. 2017, 22, 41013. [CrossRef]

64. Berg, P.J.V.D.; Bansal, R.; Daoudi, K.; Steenbergen, W.; Prakash, J. Preclinical detection of liver fibrosis using dual-modality photoacoustic/ultrasound system. Biomed. Opt. Express 2016, 7, 5081-5091. [CrossRef]

65. Jaeger, M.; Schwab, H.-M.; Almallouhi, Y.; Canal, C.; Song, M.; Sauget, V.; Sontrop, D.; Mulder, T.; Roumen, P.; Humblet, A.; et al. Deformation-Compensated Averaging for Deep-Tissue LED and Laser Diode-Based Photoacoustic Imaging Integrated with Handheld Echo Ultrasound. In LED-Based Photoacoustic Imaging; Progress in Optical Science and Photonics; Springer: Singapore, 2020; Volume 7, pp. 47-78. [CrossRef]

66. Berg, P.J.V.D.; Daoudi, K.; Steenbergen, W. Pulsed photoacoustic flow imaging with a handheld system. J. Biomed. Opt. 2016, 21, 026004. [CrossRef]

67. Upputuri, P.K.; Pramanik, M. Performance characterization of low-cost, high-speed, portable pulsed laser diode photoacoustic tomography (PLD-PAT) system. Biomed. Opt. Express 2015, 6, 4118-4129. [CrossRef]

68. Kalva, S.K.; Upputuri, P.K.; Pramanik, M. High-speed, low-cost, pulsed-laser-diode-based second-generation desktop photoacoustic tomography system. Opt. Lett. 2018, 44, 81-84. [CrossRef]

69. Rajendran, P.; Sahu, S.; Dienzo, R.A.; Pramanik, M. In vivo detection of venous sinus distension due to intracranial hypotension in small animal using pulsed-laser-diode photoacoustic tomography. J. Biophotonics 2020, 13, e201960162. [CrossRef]

70. Upputuri, P.K.; Pramanik, M. Dynamic in vivo imaging of small animal brain using pulsed laser diode-based photoacoustic tomography system. J. Biomed. Opt. 2017, 22, 1. [CrossRef]

71. Upputuri, P.K.; Das, D.; Maheshwari, M.; Yaowen, Y.; Pramanik, M. Real-time monitoring of temperature using a pulsed laser-diode-based photoacoustic system. Opt. Lett. 2020, 45, 718-721. [CrossRef]

72. Upputuri, P.K.; Pramanik, M. Fast photoacoustic imaging systems using pulsed laser diodes: A review. Biomed. Eng. Lett. 2018, 8, 167-181. [CrossRef]

73. Sivasubramanian, K.; Pramanik, M. High frame rate photoacoustic imaging at 7000 frames per second using clinical ultrasound system. Biomed. Opt. Express 2016, 7, 312-323. [CrossRef]

74. Hariri, A.; Fatima, A.; Mohammadian, N.; Mahmoodkalayeh, S.; Ansari, M.; Bely, N.; Nasiriavanaki, M. Development of low-cost photoacoustic imaging systems using very low-energy pulsed laser diodes. $J$. Biomed. Opt. 2017, 22, 75001. [CrossRef]

75. Zeng, L.; Piao, Z.; Huang, S.; Jia, W.; Chen, Z. Label-free optical-resolution photoacoustic microscopy of superficial microvasculature using a compact visible laser diode excitation. Opt. Express 2015, 23, 31026-31033. [CrossRef]

76. Wang, T.; Nandy, S.; Salehi, H.S.; Kumavor, P.D.; Zhu, Q. A low-cost photoacoustic microscopy system with a laser diode excitation. Biomed. Opt. Express 2014, 5, 3053-3058. [CrossRef]

77. Zeng, L.; Liu, G.; Yang, D.; Ji, X. Portable optical-resolution photoacoustic microscopy with a pulsed laser diode excitation. Appl. Phys. Lett. 2013, 102, 053704. [CrossRef] 
78. Erfanzadeh, M.; Kumavor, P.D.; Zhu, Q. Laser scanning laser diode photoacoustic microscopy system. Photoacoustics 2018, 9, 1-9. [CrossRef]

79. Dai, X.; Yang, H.; Jiang, H. In vivo photoacoustic imaging of vasculature with a low-cost miniature light emitting diode excitation. Opt. Lett. 2017, 42, 1456-1459. [CrossRef]

80. Steinberg, I.; Huland, D.M.; Vermesh, O.; Frostig, H.E.; Tummers, W.S.; Gambhir, S.S. Photoacoustic clinical imaging. Photoacoustics 2019, 14, 77-98. [CrossRef]

81. Kruger, R.A.; Kuzmiak, C.M.; Lam, R.B.; Reinecke, D.R.; Del Rio, S.P.; Steed, D. Dedicated 3D photoacoustic breast imaging. Med Phys. 2013, 40, 113301. [CrossRef]

82. Lin, L.; Hu, P.; Shi, J.; Appleton, C.M.; Maslov, K.; Li, L.; Zhang, R.; Wang, L.V. Single-breath-hold photoacoustic computed tomography of the breast. Nat. Commun. 2018, 9, 1-9. [CrossRef]

83. Abu Anas, E.M.; Zhang, H.K.; Kang, J.; Boctor, E. Enabling fast and high quality LED photoacoustic imaging: A recurrent neural networks based approach. Biomed. Opt. Express 2018, 9, 3852-3866. [CrossRef]

84. Mozaffarzadeh, M.; Hariri, A.; Moore, C.; Jokerst, J.V. The double-stage delay-multiply-and-sum image reconstruction method improves imaging quality in a LED-based photoacoustic array scanner. Photoacoustics 2018, 12, 22-29. [CrossRef]

85. Singh, M.K.A.; Sivasubramanian, K.; Sato, N.; Ichihashi, F.; Sankai, Y.; Xing, L. Deep learning-enhanced LED-based photoacoustic imaging. In Proceedings of the Photons Plus Ultrasound: Imaging and Sensing 2020, San Francisco, CA, USA, 17 February 2020; p. 1124038. [CrossRef]

86. Farnia, P.; Najafzadeh, E.; Hariri, A.; Lavasani, S.N.; Makkiabadi, B.; Ahmadian, A.; Jokerst, J.V. Dictionary learning technique enhances signal in LED-based photoacoustic imaging. Biomed. Opt. Express 2020, 11, 2533-2547. [CrossRef]

87. Manwar, R.; Hosseinzadeh, M.; Hariri, A.; Kratkiewicz, K.; Noei, S.; Avanaki, K. Photoacoustic Signal Enhancement: Towards Utilization of Low Energy Laser Diodes in Real-Time Photoacoustic Imaging. Sensors 2018, 18, 3498. [CrossRef]

88. Chandramoorthi, S.; Thittai, A.K. Ultrasound Receive-Side Strategies for Image Quality Enhancement in Low-Energy Illumination Based Photoacoustic Imaging. In LED-Based Photoacoustic Imaging; Progress in Optical Science and Photonics; Springer: Singapore, 2020; Volume 7, pp. 79-112. [CrossRef]

89. Chandramoorthi, S.; Thittai, A.K. Enhancing Image Quality of Photoacoustic Tomography Using Sub-Pitch Array Translation Approach: Simulation and Experimental Validation. IEEE Trans. Biomed. Eng. 2019, 66, 3543-3552. [CrossRef]

90. Sowmiya, C.; Thittai, A.K. Noise Reduction in Inherently low-SNR PLD-based PAT images. In Proceedings of the TENCON 2019-2019 IEEE Region 10 Conference (TENCON), Kochi, India, 17-20 October 2019; Institute of Electrical and Electronics Engineers (IEEE): Kochi, India, 2019; pp. 106-108.

91. Zhang, H.K. Democratizing LED-Based Photoacoustic Imaging with Adaptive Beamforming and Deep Convolutional Neural Network. In LED-Based Photoacoustic Imaging; Progress in Optical Science and Photonics; Springer: Singapore, 2020; Volume 7, pp. 183-202. [CrossRef]

92. Xia, W.; Ginsberg, Y.; West, S.J.; Nikitichev, D.I.; Ourselin, S.; David, A.L.; Desjardins, A.E. Coded excitation ultrasonic needle tracking: An in vivo study. Med Phys. 2016, 43, 4065. [CrossRef]

93. Singh, M.K.A.; Jaeger, M.; Frenz, M.; Steenbergen, W. In vivo demonstration of reflection artifact reduction in photoacoustic imaging using synthetic aperture photoacoustic-guided focused ultrasound (PAFUSion). Biomed. Opt. Express 2016, 7, 2955-2972. [CrossRef]

94. Wong, T.T.W.; Zhou, Y.; Garcia-Uribe, A.; Li, L.; Maslov, K.; Lin, L.; Wang, L.V. Use of a single xenon flash lamp for photoacoustic computed tomography of multiple-centimeter-thick biological tissue ex vivo and a whole mouse body in vivo. J. Biomed. Opt. 2016, 22, 041003. [CrossRef]

95. Wang, D.; Wang, Y.; Wang, W.; Luo, D.; Chitgupi, U.; Geng, J.; Zhou, Y.; Wang, L.; Lovell, J.F.; Xia, J. Deep tissue photoacoustic computed tomography with a fast and compact laser system. Biomed. Opt. Express 2016, 8, 112-123. [CrossRef] [PubMed]

96. Jeng, G.-S.; Li, M.-L.; Kim, M.W.; Yoon, S.J.; Pitre, J.J.; Li, D.S.; Pelivanov, I.; O’Donnell, M. Real-Time Spectroscopic Photoacoustic/Ultrasound (PAUS) Scanning with Simultaneous Fluence Compensation and Motion Correction for Quantitative Molecular Imaging; bioRxiv: New York, NY, USA, 2019.

97. Allen, T.J.; Alam, S.; Zhang, E.; Laufer, J.; Richardson, D.J.; Beard, P.C. Use of a pulsed fibre laser as an excitation source for photoacoustic tomography. In Proceedings of the Photons Plus Ultrasound: Imaging and Sensing 2011, San Francisco, CA, USA, 28 February 2011; p. 78991V. [CrossRef] 
98. Lashkari, B.; Choi, S.S.S.; Dovlo, E.; Dhody, S.; Mandelis, A. Frequency-Domain Photoacoustic Phase Spectroscopy: A Fluence-Independent Approach for Quantitative Probing of Hemoglobin Oxygen Saturation. IEEE J. Sel. Top. Quantum Electron. 2015, 22, 127-136. [CrossRef]

99. Lashkari, B.; Mandelis, A. Comparison between pulsed laser and frequency-domain photoacoustic modalities: Signal-to-noise ratio, contrast, resolution, and maximum depth detectivity. Rev. Sci. Instrum. 2011, 82, 094903. [CrossRef] [PubMed]

Publisher's Note: MDPI stays neutral with regard to jurisdictional claims in published maps and institutional affiliations.

(C) 2020 by the authors. Licensee MDPI, Basel, Switzerland. This article is an open access article distributed under the terms and conditions of the Creative Commons Attribution (CC BY) license (http://creativecommons.org/licenses/by/4.0/). 